\title{
AUBRY SETS FOR WEAKLY COUPLED SYSTEMS OF HAMILTON-JACOBI EQUATIONS
}

\author{
ANDREA DAVINI AND MAXIME ZAVIDOVIQUE
}

\begin{abstract}
We introduce a notion of Aubry set for weakly coupled systems of Hamilton-Jacobi equations on the torus and characterize it as the region where the obstruction to the existence of globally strict critical subsolutions concentrates. As in the case of a single equation, we prove the existence of critical subsolutions which are strict and smooth outside the Aubry set. This allows us to derive in a simple way a comparison result among critical sub and supersolutions with respect to their boundary data on the Aubry set, showing in particular that the latter is a uniqueness set for the critical system. We also highlight some rigidity phenomena taking place on the Aubry set.
\end{abstract}

\section{INTRODUCTION}

In this paper we will consider a weakly coupled system of Hamilton-Jacobi equations of the form

$$
H_{i}\left(x, D u_{i}\right)+\sum_{j=1}^{m} b_{i j}(x) u_{j}(x)=a \quad \text { in } \mathbb{T}^{N} \quad \text { for every } i \in\{1, \ldots, m\},
$$

where $a$ is a real constant, $H_{1}, \ldots, H_{m}$ are continuous Hamiltonians defined on the cotangent bundle of $\mathbb{T}^{N}$, convex and coercive in the momentum variable, and $B(x):=\left(b_{i j}(x)\right)$ is a continuous $m \times m$ matrix satisfying

$$
b_{i j}(x) \leqslant 0 \quad \text { for } j \neq i, \quad \sum_{j=1}^{m} b_{i j}(x)=0 \quad \text { for every } x \in \mathbb{T}^{N} \text { and } i \in\{1, \ldots, m\} .
$$

The coupling matrix will be also assumed irreducible, meaning, roughly speaking, that the coupling is non-trivial and the system cannot be split into independent subsystems. Under these assumptions it is known that there is a unique value for $a$, denoted by $c$ and termed critical in the sequel, such that the corresponding system (11) admits viscosity solutions. Critical solutions are, instead, not unique: for instance, it is easily seen that $\mathbf{u}+(\lambda, \ldots, \lambda)$ is a solution if $\mathbf{u}:=\left(u_{1}, \ldots, u_{m}\right)$ is so, for every $\lambda \in \mathbb{R}$; but in general they are not unique even up to addition of vectors of the form $(\lambda, \ldots, \lambda)$.

In this paper, we perform a qualitative analysis on the critical weakly coupled system and we investigate the kind of non-uniqueness phenomena taking place at the critical level. This is obtained by extending to the case at issue some PDE aspects of the so called weak KAM Theory, holding when there is one equation only and with no coupling. The core of our analysis is discovering that, at the critical level, a very rigid object appears, characterized as the region where the obstructions

2010 Mathematics Subject Classification. 35F21, 49L25, 37J50.

Key words and phrases. weakly coupled systems of Hamilton-Jacobi equations, viscosity solutions, weak KAM Theory. 
of strict critical subsolutions concentrates and named after Aubry in analogy with the case of a single equation. We explore the properties of this object, in particular we show that it is a uniqueness set for the critical system and we highlight some rigidity phenomena taking place on it.

Weakly coupled systems of the kind herein considered arise naturally in optimal control problems associated with randomly switching costs, where the switching is governed by specific Markov chains, see [26, 40]. In the PDE literature, they have been studied as a particular instance of monotone systems, see [15, 29, 30, and, more recently, in a series of papers addressed to study related homogenization problems in the periodic [6, 34] and stationary ergodic setting [25], and the long time behaviour of solutions to the associated evolutionary version of the system [7, 32, 33, 36]. These works can be read as a generalization of results and techniques established in the case of a single equation: see [31 for the basic homogenization results of HamiltonJacobi equations in the periodic setting, [37, 39, 1] for the subsequent extension to the stationary ergodic setting, [35, 20, 3, 38, 12, 28, for the long time convergence towards stationary solutions.

In the afore mentioned references the existence of a unique constant for which the system (11) is solvable is obtained via arguments analogous to the ones introduced in [31, in particular by making use of the so called ergodic approximation (and for this reason, the constant $c$ is also termed ergodic). A different approach, based on a second order approximation and on the new adjoint methods introduced by Evans [16] for Hamilton-Jacobi equations, is instead proposed in [5]. On the other hand, little attention has been devoted to the qualitative study of the associated critical, or ergodic, system and to the kind of nonuniqueness phenomena that occur. Some results in this direction have been obtained in [7], where the existence of a uniqueness set for the critical system is derived in the specific case ${ }^{1}$ when the Hamiltonians are of the form

$$
H_{i}(x, p):=F_{i}(x, p)-V_{i}(x) \quad \text { for every }(x, p) \in \mathbb{T}^{N} \times \mathbb{R}^{N} \text { and } i \in\{1, \ldots, m\},
$$

with $V_{i}$ non-negative functions satisfying $\bigcap_{i=1}^{m} V_{i}^{-1}(\{0\}) \neq \varnothing$, and $F_{i}$ convex and coercive in $p$, and such that

$$
F_{i}(x, p) \geqslant F_{i}(x, 0)=0 \quad \text { for every }(x, p) \in \mathbb{T}^{N} \times \mathbb{R}^{N} .
$$

For the analysis performed in this paper we employ techniques borrowed from the weak KAM Theory. This theory, developed by Fathi to study the dynamics of Hamiltonian systems, has revealed to be a powerful tool for a fine qualitative analysis of the stationary critical equation associated with a Tonelli Hamiltonian. Fathi discovered in fact [17, 18, 19, 20] that the Aubry set appears as the dynamical part of the set of points where all the critical viscosity subsolutions are smooth; moreover, it is a uniqueness set for the critical equation. His approach relies on a characterization of the critical solutions in terms of variational formulae. We refer the reader to [22] for an expository presentation of the theory for Tonelli Hamiltonians and to [21] for the extension of its PDE aspects to less regular cases.

The generalization of the weak KAM Theory proposed in this paper was however nontrivial. A first serious difficulty is that we could not find suitable variational formulae to express the solutions of the system. We have overcome this problem

\footnotetext{
${ }^{1}$ The conditions on the coupling matrix are instead more general, in the sense that the sums of the elements on each row of $B(x)$ are not required to vanish identically on the whole torus, but only on a part of it.
} 
by choosing a more PDE oriented viewpoint to weak KAM Theory and by making use of viscosity solution techniques. A second issue is that, to extend this approach to the case at issue, we needed to better understand the different structure of the problem and the role of the coupling. This has been achieved by recognizing and exploiting the vectorial nature of the system.

The starting point of our study relies on a different definition of the critical value $c$, given as the minimal $a \in \mathbb{R}$ for which the corresponding weakly coupled system admits viscosity subsolutions. We subsequently show that the associated critical weakly coupled system is the unique among those of the family (11) that admits viscosity solutions. This characterization of the critical value is not new, see for instance [32, but our approach to the existence part is different: it is based on a fixed point argument in the spirit of 22 and on the properties of the semigroup associated with the evolutionary version of the system (11).

We then study the corresponding critical weakly coupled system and show that the obstruction to the existence of globally strict subsolutions is not spread indistinctly on the torus, but concentrates on a closed set $\mathcal{A}$, that we call Aubry set in analogy to the case of a single equation. Moreover, we show the existence of critical subsolutions smooth and strict outside the Aubry set. This allows us to derive in a simple way a comparison result among critical sub and supersolutions satisfying suitable "boundary" conditions on $\mathcal{A}$, see Theorem 5.5. This generalizes to our setting Theorem 3.3 in [7. In particular, we infer that the Aubry set is a uniqueness set for the critical system. We furthermore show that the trace of any critical subsolution on $\mathcal{A}$ can be extended on the whole torus in such a way that the output is a critical solution, see Theorem 5.7 .

Our study highlights some rigidity phenomena taking place on the Aubry set. First, we show that any pair of critical subsolutions differ, at each point $y$ of $\mathcal{A}$, by a vector of the form $k(1,1, \ldots, 1)$, see Theorem [5.1. In the particular case when there exists a critical subsolution of the kind $(v(x), v(x), \ldots, v(x))$, we infer that any other critical subsolution is of this form on $\mathcal{A}$. This accounts for the kind of symmetries already observed in [7] for the particular class of Hamiltonians therein considered, see Section 6.1 for more details.

A second rigidity phenomenon that we point out is when the Hamiltonians are additionally assumed strictly convex in the momentum: in this case we prove that, at any point of the Aubry set, the intersection of the reachable gradients of all the critical subsolutions is always nonempty, see Proposition 4.4. This can be regarded as a weak version of a result holding in the scalar case: under suitable regularity assumptions on the Hamiltonian, it is in fact well known that critical subsolutions are all differentiable on the Aubry set and have the same gradient, see [22, 23, 24].

We end our study by presenting some situations where more explicit results can be obtained for the critical value and for the Aubry set, see Section 6. In the first example, we focus on the setting studied in [7] and we show that our notion of Aubry set is consistent with the one therein given. Next we show how such analysis can be extended to a variant of this model considered in [36]. For this, we make use of a general result on weakly coupled systems with constant coupling matrices which is of independent interest, see Proposition 6.2. The last example contains, as a particular instance, the case when the Hamiltonians are all equal, say to $H$. In this specific situation, we show that the critical value and the Aubry set of the weakly coupled system agree with the critical value and the Aubry set of $H$. We furthermore show that the solutions of the critical system are all of the form $u(x)(1, \ldots, 1)$, where $u$ is 
a critical solution for $H$, thus showing that, as far as critical solutions of the system are concerned, the coupling is not playing any effective role.

This paper is organized as follows. In Section 1.1 we fix the notations and assumptions, and we give a brief overview of existing results on weakly coupled systems. Section 2 is devoted to the definition of the critical value and to the study of its main properties. Some proofs are postponed to the Appendix A. In Section 3 we give the definition of Aubry set and explore its properties. The first part of Section 4 is devoted to the regularization of subsolutions outside of the Aubry set, while in the second part we prove the rigidity phenomenon enjoyed by the reachable gradients of the critical subsolutions previously described. The other rigidity phenomenon is instead proved at the beginning of Section 5 and some consequences are drawn. In the remainder of the section we prove the comparison principle, Theorem [5.5, and we show how the trace of a critical subsolution on the Aubry set can be extended to the whole torus to produce a critical solution, see Theorem [5.7. In Section 6] we illustrate our theory on some examples.

ACKNowledGements. - This research was initiated while the first author was visiting the Institut de Mathématiques de Jussieu, Université Pierre et Marie Curie, Paris, that he acknowledges for the kind hospitality. The first author has been supported by Sapienza Università di Roma through the Research Project 2011 Analisi ed approssimazione di modelli differenziali nonlineari in fluidodinamica e scienza dei materiali. The second author is partially supported by ANR-12-BLAN-WKBHJ.

\section{Preliminaries}

1.1. Notations. Throughout the paper, we will denote by $\mathbb{T}^{N}=\mathbb{R}^{N} / \mathbb{Z}^{N}$ the $N$ dimensional flat torus, where $N$ is an integer number. The scalar product in $\mathbb{R}^{N}$ will be denoted by $\langle\cdot, \cdot\rangle$, while the symbol $|\cdot|$ stands for the Euclidean norm. Note that the latter induces a distance on $\mathbb{T}^{N}$, denoted by $d(\cdot, \cdot)$, defined as

$$
d(x, y):=\min _{\kappa \in \mathbb{Z}^{N}}|x-y+\kappa| \quad \text { for every } x, y \in \mathbb{T}^{N} .
$$

More generally, if $X \subset \mathbb{T}^{N}$ is a set, we will denote by $d(x, X):=\inf _{y \in X} d(x, y)$ the distance from a point $x \in \mathbb{T}^{N}$ to $X$. We will denote by $B_{R}\left(x_{0}\right)$ and $B_{R}$ the closed balls in $\mathbb{T}^{N}$ of radius $R$ centered at $x_{0}$ and 0 , respectively.

With the symbol $\mathbb{R}_{+}$we will refer to the set of nonnegative real numbers. We say that a property holds almost everywhere (a.e. for short) in a subset $E$ of $\mathbb{T}^{N}$ if it holds up to a negligible subset of $E$, i.e. a subset of zero $N$-dimensional Lebesgue measure.

By modulus we mean a nondecreasing function from $\mathbb{R}_{+}$to $\mathbb{R}_{+}$, vanishing and continuous at 0 . A function $g: \mathbb{R}_{+} \rightarrow \mathbb{R}$ will be termed coercive if $\lim _{h \rightarrow+\infty} g(h)=+\infty$.

We will say that $\left(\rho_{n}\right)_{n}$ is a sequence of standard mollifiers if $\rho_{n}(x):=n^{N} \rho(n x)$ in $\mathbb{R}^{N}$ for each $n \in \mathbb{N}$, where $\rho$ is a smooth, non-negative function on $\mathbb{R}^{N}$, supported in $B_{1}$ and such that its integral over $\mathbb{R}^{N}$ is equal to 1 .

Given a continuous function $u$ on $\mathbb{T}^{N}$, we will call subtangent (respectively, supertangent) of $u$ at $x_{0}$ a function $\phi$ of class $C^{1}$ in a neighborhood $U$ of $x_{0}$ such that $u-\phi$ has a local minimum (resp., maximum) at $x_{0}$. Its gradient $D \phi\left(x_{0}\right)$ will be 
called a subdifferential (resp. superdifferential) of $u$ at $x_{0}$. The set of sub and superdifferentials of $u$ at $x_{0}$ will be denoted $D^{-} u\left(x_{0}\right)$ and $D^{+} u\left(x_{0}\right)$, respectively. The function $\phi$ will be furthermore termed strict subtangent (resp., strict supertangent) if $u-\phi$ has a strict local minimum (resp., maximum) at $x_{0}$. Any subtangent (resp., supertangent) $\phi$ of $u$ can be always assumed strict at $x_{0}$ without affecting $D \phi\left(x_{0}\right)$ by possibly replacing it with $\phi-d^{2}\left(x_{0}, \cdot\right)\left(\right.$ resp. $\left.\phi+d^{2}\left(x_{0}, \cdot\right)\right)$. We recall that $u$ is differentiable at $x_{0}$ if and only if $D^{+} u\left(x_{0}\right)$ and $D^{-} u\left(x_{0}\right)$ are both nonempty. In this instance, $D^{+} u\left(x_{0}\right)=D^{-} u\left(x_{0}\right)=\left\{D u\left(x_{0}\right)\right\}$. We refer the reader to [8] for the proofs.

When $u$ is locally Lipschitz in $\mathbb{T}^{N}$, we will denote by $\partial^{*} u\left(x_{0}\right)$ the set of reachable gradients of $u$ at $x_{0}$, that is the set

$$
\partial^{*} u\left(x_{0}\right)=\left\{\lim _{n} D u\left(x_{n}\right): u \text { is differentiable at } x_{n}, x_{n} \rightarrow x_{0}\right\},
$$

while the Clarke's generalized gradient $\partial^{c} u\left(x_{0}\right)$ is the closed convex hull of $\partial^{*} u\left(x_{0}\right)$. The set $\partial^{c} u\left(x_{0}\right)$ contains both $D^{+} u\left(x_{0}\right)$ and $D^{-} u\left(x_{0}\right)$, in particular $D u\left(x_{0}\right) \in$ $\partial^{c} u\left(x_{0}\right)$ at any differentiability point $x_{0}$ of $u$. We refer the reader to [10] for a detailed treatment of the subject.

We will denote by $\|g\|_{\infty}$ the usual $L^{\infty}$-norm of $g$, where the latter is a measurable real function defined on $\mathbb{T}^{N}$. We will write $g_{n} \rightrightarrows g$ in $\mathbb{T}^{N}$ to mean that the sequence of functions $\left(g_{n}\right)_{n}$ uniformly converges to $g$ in $\mathbb{T}^{N}$, i.e. $\left\|g_{n}-g\right\|_{\infty} \rightarrow 0$. We will denote by $\left(\mathrm{C}\left(\mathbb{T}^{N}\right)\right)^{m}$ the Banach space of continuous functions $\mathbf{u}=\left(u_{1}, \ldots, u_{m}\right)^{T}$ from $\mathbb{T}^{N}$ to $\mathbb{R}^{m}$, endowed with the norm

$$
\|\mathbf{u}-\mathbf{v}\|_{\infty}=\max _{1 \leqslant i \leqslant m}\left\|u_{i}-v_{i}\right\|_{\infty}, \quad \mathbf{u}, \mathbf{v} \in\left(\mathrm{C}\left(\mathbb{T}^{N}\right)\right)^{m} .
$$

We will write $\mathbf{u}^{n} \rightrightarrows \mathbf{u}$ in $\mathbb{T}^{N}$ to mean that $\left\|\mathbf{u}^{n}-\mathbf{u}\right\|_{\infty} \rightarrow 0$. A function $\mathbf{u} \in\left(\mathrm{C}\left(\mathbb{T}^{N}\right)\right)^{m}$ will be termed Lipschitz continuous if each of its components is $\kappa$-Lipschitz continuous, for some $\kappa>0$. Such a constant $\kappa$ will be called a Lipschitz constant for $\mathbf{u}$. The space of all such functions will be denoted by $\left(\operatorname{Lip}\left(\mathbb{T}^{N}\right)\right)^{m}$.

We will denote by $\mathbb{1}=(1, \cdots, 1)^{T}$ the vector of $\mathbb{R}^{m}$ having all components equal to 1 , where the upper-script symbol $T$ stands for the transpose. We consider the following partial relations between elements $\mathbf{a}, \mathbf{b} \in \mathbb{R}^{m}: \mathbf{a} \leqslant \mathbf{b}$ (respectively, $\mathbf{a}<\mathbf{b}$ ) if $a_{i} \leqslant b_{i}$ (resp., $<$ ) for every $i \in\{1, \ldots, m\}$. Given two functions $\mathbf{u}, \mathbf{v}: \mathbb{T}^{N} \rightarrow \mathbb{R}^{m}$, we will write $\mathbf{u} \leqslant \mathbf{v}$ in $\mathbb{T}^{N}$ (respectively, $<$ ) to mean that $\mathbf{u}(x) \leqslant \mathbf{v}(x)$ (resp., $\mathbf{u}(x)<\mathbf{v}(x))$ for every $x \in \mathbb{T}^{N}$.

1.2. Linear algebra. Here we briefly present some elementary linear algebraic results concerning coupling matrices.

Definition 1.1. Let $B=\left(b_{i j}\right)_{i, j}$ be a $m \times m$-matrix.

(i) We say that $B$ is a coupling matrix if it satisfies the following conditions:

$$
b_{i j} \leqslant 0 \text { for } j \neq i, \quad \sum_{j=1}^{m} b_{i j} \geqslant 0 \quad \text { for any } i \in\{1, \ldots, m\} .
$$

It is additionally termed degenerate if

$$
\sum_{j=1}^{m} b_{i j}=0 \quad \text { for any } i=1, \ldots, m \text {. }
$$


(ii) We say that $B$ is irreducible if for every subset $\mathcal{I} \subsetneq\{1, \ldots, m\}$ there exist $i \in \mathcal{I}$ and $j \notin \mathcal{I}$ such that $b_{i j} \neq 0$.

When a coupling matrix is also irreducible, further information can be derived on its elements. We have

Proposition 1.2. Let $B=\left(b_{i j}\right)_{i, j}$ be an irreducible $m \times m$ coupling matrix. Then $b_{i i}>0 \quad$ for every $i \in\{1, \ldots, m\}$.

Proof. Indeed, if $b_{i_{0} i_{0}}=0$ for some $i_{0} \in\{1, \ldots, m\}$, condition (C) would imply $b_{i_{0} j}=0$ for every $j \in\{1, \ldots, m\}$, in contradiction with the fact that $B$ is irreducible.

The following invertibility criterion holds:

Proposition 1.3. Let $B=\left(b_{i j}\right)_{i, j}$ be an $m \times m$ irreducible coupling matrix. Then

(i) $\operatorname{Ker}(B) \subseteq \operatorname{span}\left\{(1, \ldots, 1)^{T}\right\}=\mathbb{R} \mathbb{1}$;

(ii) $\operatorname{Ker}(B)=\operatorname{span}\left\{(1, \ldots, 1)^{T}\right\}=\mathbb{R} \mathbb{1} \quad$ if and only if $B$ is degenerate.

In particular, $B$ is invertible if and only if

$$
\sum_{j=1}^{m} b_{i j}>0 \quad \text { for some } i \in\{1, \ldots, m\} .
$$

Proof. We first remark that, by assumption (C),

$$
b_{i i} \geqslant \sum_{j \neq i}\left|b_{i j}\right| \quad \text { for every } i \in\{1, \ldots, m\} .
$$

Let us prove $(i)$. Let $\mathbf{v}=\left(v_{1}, \ldots, v_{m}\right)^{T} \in \operatorname{Ker}(B)$ and set

$$
\mathcal{I}=\left\{i \in\{1, \ldots, m\}: v_{i}=\max \left\{v_{1}, \ldots, v_{m}\right\}\right\} .
$$

We claim that $\mathcal{I}=\{1, \ldots, m\}$. Indeed, if this were not the case, by the irreducible character of $B$ there would exist $i \in \mathcal{I}$ and $k \notin \mathcal{I}$ such that $b_{i k} \neq 0$. Since $B \mathbf{v}=0$, we would get in particular

$$
b_{i i} v_{i}=\sum_{j \neq i} v_{j}\left|b_{i j}\right| \leqslant v_{i} \sum_{j \neq i}\left|b_{i j}\right| \leqslant v_{i} b_{i i}
$$

Then the inequalities must be equalities. We infer

$$
v_{j}\left|b_{i j}\right|=v_{i}\left|b_{i j}\right| \quad \text { for every } j \neq i,
$$

in particular $v_{k}=v_{i}=\max \left\{v_{1}, \ldots, v_{m}\right\}$, yielding that $k$ belongs to $\mathcal{I}$, a contradiction.

The remainder of the statement trivially follows from item (i).

The following proposition gives an obstruction to being in the image of a degenerate coupling matrix.

Proposition 1.4. Let $B$ a coupling and degenerate $m \times m$ matrix. If $\mathbf{a}=\left(a_{1}, \ldots, a_{m}\right)^{T}$ satisfies $a_{i}>0$ for every $i \in\{1, \ldots, m\}$, then $\mathbf{a} \notin \operatorname{Im}(B)$. 
Proof. Let us assume by contradiction that there exists $\mathbf{v}=\left(v_{1}, \ldots, v_{m}\right)^{T}$ such that

$$
B \mathbf{v}=\mathbf{a}
$$

Let $v_{k}=\min \left\{v_{1}, \ldots, v_{m}\right\}$. We have

$$
a_{k}=\sum_{j=1}^{m} b_{k j} v_{j} \leqslant \sum_{j=1}^{m} b_{k j} v_{k}=0,
$$

in contradiction with the hypothesis $a_{k}>0$.

1.3. Weakly coupled systems. Throughout the paper, we will call convex Hamiltonian a function $H$ satisfying the following set of assuptions:

$$
\begin{aligned}
& H: \mathbb{T}^{N} \times \mathbb{R}^{N} \rightarrow \mathbb{R} \quad \text { is continuous; } \\
& p \mapsto H(x, p) \quad \text { is convex on } \mathbb{R}^{N} \text { for any } x \in \mathbb{T}^{N} ;
\end{aligned}
$$

there exist two coercive functions $\alpha, \beta: \mathbb{R}_{+} \rightarrow \mathbb{R}$ such that

$$
\alpha(|p|) \leqslant H(x, p) \leqslant \beta(|p|) \quad \text { for all }(x, p) \in \mathbb{T}^{N} \times \mathbb{R}^{N} .
$$

The Hamiltonian $H$ will be termed strictly convex if it additionally satisfies the following stronger assumption:

$(\mathrm{H} 2)^{\prime} \quad p \mapsto H(x, p) \quad$ is strictly convex on $\mathbb{R}^{N}$ for any $x \in \mathbb{T}^{N}$.

Moreover, we will denote by $B(x)=\left(b_{i j}(x)\right)_{i, j}$ an $m \times m$-matrix with continuous coefficients $b_{i j}(x)$ on $\mathbb{T}^{N}$. If not otherwise stated, the following hypotheses will be always assumed:

(B1) $\quad B(x)$ is an irreducible coupling matrix for every $x \in \mathbb{T}^{N}$;

$$
B(x) \text { is degenerate for every } x \in \mathbb{T}^{N} \text {. }
$$

Let $H_{1}(x, p), \ldots, H_{m}(x, p)$ be convex Hamiltonians, i.e. functions satisfying conditions (H1)-(H3). We are interested in weakly coupled systems of the form

$$
H_{i}\left(x, D u_{i}\right)+(B(x) \mathbf{u}(x))_{i}=a_{i} \quad \text { in } \mathbb{T}^{N} \quad \text { for every } i \in\{1, \ldots, m\},
$$

for some constant vector $\mathbf{a}=\left(a_{1}, \ldots, a_{m}\right)^{T}$, where $\mathbf{u}(x)=\left(u_{1}(x), \ldots, u_{m}(x)\right)^{T}$ and $(B(x) \mathbf{u}(x))_{i}$ denotes the $i$-th component of the vector $B(x) \mathbf{u}(x)$, i.e.

$$
(B(x) \mathbf{u}(x))_{i}=\sum_{j=1}^{m} b_{i j}(x) u_{j}(x) .
$$

Remark 1.5. The weakly coupled system (1.2) is a particular type of monotone system, i.e. a system of the form

$$
G_{i}\left(x, u_{1}(x), \ldots, u_{m}(x), D u_{i}\right)=0 \quad \text { in } \mathbb{T}^{N} \quad \text { for every } i \in\{1, \ldots, m\},
$$

where suitable monotonicity conditions with respect to the $u_{j}$-variables are assumed on the functions $G_{i}$, see $[6,15,27,29,30]$. In the specific case considered in this paper, the conditions assumed on the coupling matrix imply, in particular, that each function $G_{i}$ is strictly increasing in $u_{i}$ and non-increasing in $u_{j}$ for every $j \neq i$. This kind of monotonicity will be exploited in many points of the paper. 
Let $\mathbf{u} \in\left(\mathrm{C}\left(\mathbb{T}^{N}\right)\right)^{m}$. We will say that $\mathbf{u}$ is a viscosity subsolution of (1.2) if the following inequality holds for every $(x, i) \in \mathbb{T}^{N} \times\{1, \ldots, m\}$

$$
H_{i}(x, p)+(B(x) \mathbf{u}(x))_{i} \leqslant a_{i} \text { for every } p \in D^{+} u_{i}(x) .
$$

We will say that $\mathbf{u}$ is a viscosity supersolution of (1.2) if the following inequality holds for every $(x, i) \in \mathbb{T}^{N} \times\{1, \ldots, m\}$

$$
H_{i}(x, p)+(B(x) \mathbf{u}(x))_{i} \geqslant a_{i} \quad \text { for every } p \in D^{-} u_{i}(x) .
$$

We will say that $\mathbf{u}$ is a viscosity solution if it is both a sub and a supersolution. In the sequel, solutions, subsolutions and supersolutions will be always meant in the viscosity sense, hence the adjective viscosity will be omitted.

Due to the convexity of the Hamitonian $H_{i}$, the following equivalences hold:

Proposition 1.6. Let $a \in \mathbb{R}, i \in\{1, \ldots, m\}$ and $\mathbf{u} \in\left(\operatorname{Lip}\left(\mathbb{T}^{N}\right)\right)^{m}$. The following facts are equivalent:

$$
\begin{array}{ll}
H_{i}(x, p)+(B(x) \mathbf{u}(x))_{i} \leqslant a & \text { for every } p \in D^{+} u_{i}(x) \text { and } x \in \mathbb{T}^{N} \\
H_{i}(x, p)+(B(x) \mathbf{u}(x))_{i} \leqslant a & \text { for every } p \in D^{-} u_{i}(x) \text { and } x \in \mathbb{T}^{N} \\
H_{i}(x, p)+(B(x) \mathbf{u}(x))_{i} \leqslant a & \text { for every } p \in \partial^{c} u_{i}(x) \text { and } x \in \mathbb{T}^{N} \\
H_{i}\left(x, D u_{i}(x)\right)+(B(x) \mathbf{u}(x))_{i} \leqslant a & \text { for a.e. } x \in \mathbb{T}^{N} .
\end{array}
$$

Next, we state a proposition that will be needed in the sequel, see also [15, 30 , [27, 29] for similar results.

Proposition 1.7. Let $\mathcal{F}$ be a subset of $\left(\mathrm{C}\left(\mathbb{T}^{N}\right)\right)^{m}$ and define the functions $\underline{\mathbf{u}}, \overline{\mathbf{u}}$ on $\mathbb{T}^{N}$ by setting:

$$
\underline{u}_{i}(x)=\inf _{\mathbf{u} \in \mathcal{F}} u_{i}(x), \quad \bar{u}_{i}(x)=\sup _{\mathbf{u} \in \mathcal{F}} u_{i}(x) \quad \text { for every } x \in \mathbb{T}^{N} \text { and } i \in\{1, \ldots, m\} .
$$

Assume that $\underline{\mathbf{u}}$ and $\overline{\mathbf{u}}$ belong to $\left(\mathrm{C}\left(\mathbb{T}^{N}\right)\right)^{m}$ and let $\mathbf{a} \in \mathbb{R}^{m}$. Then:

(i) if every $\mathbf{u} \in \mathcal{F}$ is a subsolution of (1.2), then $\overline{\mathbf{u}}$ is a subsolution of (1.2);

(ii) if every $\mathbf{u} \in \mathcal{F}$ is a supersolution of (1.2), then $\underline{\mathbf{u}}$ is a supersolution of (1.2) .

The previous proposition is analogous to a well known fact for scalar HamiltonJacobi equations, see for instance Section 2.6 in [2]. The proof can be easily recovered by arguing similarly and by exploiting the monotonicity of the system.

We will be also interested in the evolutionary counterpart of (1.2), i.e. the system

$$
\frac{\partial u_{i}}{\partial t}+H_{i}\left(x, D_{x} u_{i}\right)+(B(x) \mathbf{u}(t, x))_{i}=0 \quad \text { in }(0,+\infty) \times \mathbb{T}^{N} \quad \forall i \in\{1, \ldots, m\},
$$

where we have denoted by $\mathbf{u}(t, x)=\left(u_{1}(t, x), \ldots, u_{m}(t, x)\right)^{T}$.

The following comparison result holds, see for instance [6] for a proof.

Proposition 1.8. Let $T>0$ and $\mathbf{v}, \mathbf{u} \in\left(\operatorname{Lip}\left([0, T] \times \mathbb{T}^{N}\right)\right)^{m}$ be, respectively, a sub and a supersolution of (1.3). Then, for every $i \in\{1, \ldots, m\}$,

$$
v_{i}(t, x)-u_{i}(t, x) \leqslant \max _{1 \leqslant i \leqslant m} \max _{\mathbb{T}^{N}}\left(v_{i}(0, \cdot)-u_{i}(0, \cdot)\right), \quad(t, x) \in[0, T] \times \mathbb{T}^{N} .
$$

By making use of this proposition and of Perron's method, it is then easy to prove the following 
Proposition 1.9. Let $\mathbf{u}_{0} \in\left(\operatorname{Lip}\left(\mathbb{T}^{N}\right)\right)^{m}$. Then there exists a unique function $\mathbf{u}(t, x)$ in $\left(\operatorname{Lip}\left(\mathbb{R}_{+} \times \mathbb{T}^{N}\right)\right)^{m}$ that solves the system (1.3) subject to the initial condition $\mathbf{u}(0, x)=\mathbf{u}_{0}(x)$ in $\mathbb{T}^{N}$. Moreover, the Lipschitz constant of $\mathbf{u}(t, x)$ in $\mathbb{R}_{+} \times \mathbb{T}^{N}$ only depends on the Hamiltonians $H_{1}, \ldots, H_{m}$ and on the Lipschitz constant of $\mathbf{u}_{0}$.

We will denote by $\mathcal{S}(t) \mathbf{u}_{0}(x)$ the solution $\mathbf{u}(t, x)$ of (1.3) with initial datum $\mathbf{u}_{0}$. This defines, for every $t>0$, a map

$$
\mathcal{S}(t):\left(\operatorname{Lip}\left(\mathbb{T}^{N}\right)\right)^{m} \rightarrow\left(\operatorname{Lip}\left(\mathbb{T}^{N}\right)\right)^{m}
$$

We summarize in the next proposition the properties enjoyed by such maps, which come as an easy application of the above results.

Proposition 1.10. For every $t, s>0$ and $\mathbf{u}, \mathbf{v} \in\left(\operatorname{Lip}\left(\mathbb{T}^{N}\right)\right)^{m}$ we have:

(i) (Semigroup property) $\mathcal{S}(s)(\mathcal{S}(t) \mathbf{u})=\mathcal{S}(t+s) \mathbf{u} \quad$ in $\mathbb{T}^{N}$;

(ii) (Monotonicity) if $\mathbf{v} \leqslant \mathbf{u} \quad$ in $\mathbb{T}^{N}$, then $\mathcal{S}(t) \mathbf{v} \leqslant \mathcal{S}(t) \mathbf{u} \quad$ in $\mathbb{T}^{N}$;

(iii) (Non-expansiveness property) $\|\mathcal{S}(t) \mathbf{v}-\mathcal{S}(t) \mathbf{u}\|_{\infty} \leqslant\|\mathbf{v}-\mathbf{u}\|_{\infty}$;

(iv) for every $a \in \mathbb{R}, \quad \mathcal{S}(t)(\mathbf{u}+a \mathbb{1})=\mathcal{S}(t) \mathbf{u}+a \mathbb{1} \quad$ in $\mathbb{T}^{N}$.

The fact that the coupling matrix $B(x)$ is everywhere degenerate is crucial for assertion (iv).

\section{The CRitical value}

The purpose of this section is to define the notion of critical value for weakly coupled systems and to prove some relevant properties of the corresponding critical system.

We start by proving some a priori estimates for the subsolutions of a weakly coupled system of the form (1.2). The following notation will be assumed throughout the section:

$$
\mu_{i}=\min _{(x, p)} H_{i}(x, p) \quad \text { for each } i \in\{1, \ldots, m\}, \quad \mu=\min _{i \in\{1, \ldots, m\}} \mu_{i} .
$$

Proposition 2.1. Let $\mathbf{a}=\left(a_{1}, \ldots, a_{m}\right)^{T} \in \mathbb{R}^{m}$ and $\mathbf{u} \in\left(\mathrm{C}\left(\mathbb{T}^{N}\right)\right)^{m}$ such that

$$
(B(x) \mathbf{u}(x))_{i} \leqslant a_{i} \quad \text { for every } x \in \mathbb{T}^{N} \text { and } i \in\{1, \ldots, m\} .
$$

Then there exists a constant $M_{\mathbf{a}}$ only depending on $\mathbf{a}$ and $B(x)$ such that

$$
\left\|u_{i}-u_{j}\right\|_{\infty} \leqslant M_{\mathbf{a}} \quad \text { for every } i, j \in\{1, \ldots, m\}
$$

(ii) $\left|(B(x) \mathbf{u}(x))_{i}\right| \leqslant M_{\mathbf{a}} \quad$ for every $x \in \mathbb{T}^{N}$ and $i \in\{1, \ldots, m\}$.

Proof. It suffices to prove the assertion for $\mathbf{a}=a \mathbb{1}$. Let us set

$$
\beta_{\star}=\min _{1 \leqslant i \leqslant m} \min _{x \in \mathbb{T}^{N}} b_{i i}(x), \quad \beta^{\star}=\max _{1 \leqslant i, j \leqslant m} \max _{x \in \mathbb{T}^{N}}\left|b_{i j}(x)\right| .
$$

Such quantities are finite valued. Moreover, $\beta_{\star}$ is strictly positive in view of Proposition 1.2 and of the fact that $B(x)$ is, for every $x \in \mathbb{T}^{N}$, an irreducible coupling matrix with continuous coefficients.

Let us now fix $x \in \mathbb{T}^{N}$ and assume, without any loss of generality,

$$
u_{1}(x) \leqslant u_{2}(x) \leqslant \cdots \leqslant u_{m}(x) .
$$


First notice that, by subtracting $\sum_{j=1}^{m} b_{m j}(x) u_{m}(x)=0$ from both sides of equation (2.1) with $i=m$, one gets

$$
\sum_{j \neq m}-b_{m j}(x)\left(u_{m}(x)-u_{j}(x)\right) \leqslant a
$$

yielding

$$
\left(u_{m}(x)-\max _{j \neq m} u_{j}(x)\right) \sum_{j \neq m}-b_{m j}(x) \leqslant a .
$$

By exploiting (2.2) and the degenerate character of the matrix $B(x)$ we get

$$
0 \leqslant u_{m}(x)-u_{m-1}(x) \leqslant \frac{a}{b_{m m}(x)} \leqslant \frac{a}{\beta_{\star}} .
$$

This proves assertion (i) when $m=2$. To prove it in the general case, we argue by induction: we assume the result true for $m$ and we prove it for $m+1$. To this aim, we restate equation (2.1) as

$$
\sum_{j=1}^{m-1} b_{i j}(x) u_{j}(x)+\left(b_{i m}(x)+b_{i m+1}(x)\right) u_{m}(x)+b_{i m+1}(x)\left(u_{m+1}(x)-u_{m}(x)\right) \leqslant a,
$$

then we exploit (2.3) to get

$$
\sum_{j=1}^{m-1} b_{i j}(x) u_{j}(x)+\left(b_{i m}(x)+b_{i m+1}(x)\right) u_{m}(x) \leqslant a\left(1+\frac{\beta^{\star}}{\beta_{\star}}\right)
$$

for every $i \in\{1, \ldots, m+1\}$. The irreducible character of $B(x)$ applied to the set $\mathcal{I}=\{m, m+1\}$ implies that

$$
b_{i m}(x)+b_{i m+1}(x)>0
$$

for either $i=m$ or $i=m+1$, let us say $i=m$ for definitiveness. Assertion (i) now follows by applying the induction hypothesis to the system given by (2.4) with $i$ varying in $\{1, \ldots, m\}$, the corresponding coupling matrix being still irreducible and degenerate.

To prove (ii) it suffices to note that, for every $i \in\{1, \ldots, m\}$,

$$
\begin{aligned}
-(B(x) \mathbf{u}(x))_{i} & =-b_{i i}(x) u_{i}(x)+\sum_{j \neq i}\left(-b_{i j}(x)\right) u_{j}(x) \\
& \leqslant-b_{i i}(x) u_{i}(x)+\sum_{j \neq i}-b_{i j}(x)\left(u_{i}(x)+\left\|u_{i}-u_{j}\right\|_{\infty}\right) \\
& \quad \leqslant(m-1) \beta^{\star}\left\|u_{i}-u_{j}\right\|_{\infty},
\end{aligned}
$$

and the assertion follows from (i) and from hypothesis (2.1).

As a consequence, we derive the following result:

Proposition 2.2. Let $\mathbf{u}=\left(u_{1}, \ldots, u_{m}\right)^{T} \in\left(\mathrm{C}\left(\mathbb{T}^{N}\right)\right)^{m}$ be a subsolution of (1.2) for some $\mathbf{a} \in \mathbb{R}^{m}$. Then there exist constants $C_{\mathbf{a}}$ and $\kappa_{\mathbf{a}}$, only depending on $\mathbf{a}$, on the Hamiltonians $H_{1}, \ldots, H_{m}$ and on the coupling matrix $B(x)$, such that

(i) $\left\|u_{i}-u_{j}\right\|_{\infty} \leqslant C_{\mathbf{a}} \quad$ for every $i, j \in\{1, \ldots, m\}$;

(ii) $\mathbf{u}$ is $\kappa_{\mathbf{a}}$-Lipschitz continuous in $\mathbb{T}^{N}$. 
Proof. For each $i \in\{1, \ldots, m\}$, the following inequalities hold in the viscosity sense:

$$
\mu+(B(x) \mathbf{u}(x))_{i} \leqslant H_{i}\left(x, D u_{i}\right)+(B(x) \mathbf{u}(x))_{i} \leqslant a_{i} \quad \text { in } \mathbb{T}^{N},
$$

yielding

$$
(B(x) \mathbf{u}(x))_{i} \leqslant a_{i}-\mu \quad \text { for every } x \in \mathbb{T}^{N} .
$$

In view of Proposition 2.1 we get (i) and

$$
\left|(B(x) \mathbf{u}(x))_{i}\right| \leqslant M_{\mathbf{a}} \quad \text { for every } x \in \mathbb{T}^{N} .
$$

Plugging this inequality in (1.2) we derive that $u_{i}$ is a viscosity subsolution of

$$
H_{i}\left(x, D u_{i}\right) \leqslant a_{i}+M_{\mathbf{a}} \quad \text { in } \mathbb{T}^{N}
$$

and assertion (ii) follows as well via a standard argument that exploits the coercivity of $H_{i}(x, p)$ in $p$, see for instance [2].

Next, we establish a remarkable property of weakly coupled systems.

Proposition 2.3. Assume that $\mathbf{v}, \mathbf{u} \in\left(\mathrm{C}\left(\mathbb{T}^{N}\right)\right)^{m}$ are, respectively a sub and a supersolution of the weakly coupled system (1.2) for some $\mathbf{a} \in \mathbb{R}^{m}$. Let $x_{0} \in \mathbb{T}^{N}$ be such that

$$
v_{i}\left(x_{0}\right)-u_{i}\left(x_{0}\right)=M:=\max _{1 \leqslant i \leqslant m} \max _{\mathbb{T}^{N}}\left(v_{i}-u_{i}\right) \quad \text { for some } i \in\{1, \ldots, m\} .
$$

Then $\mathbf{v}\left(x_{0}\right)=\mathbf{u}\left(x_{0}\right)+M \mathbb{1}$.

Proof. In view of Proposition 2.2, we know that $\mathbf{v}$ is Lipschitz continuous. Set

$$
\mathcal{I}=\left\{i \in\{1, \ldots, m\}:\left(v_{i}\left(x_{0}\right)-u_{i}\left(x_{0}\right)\right)=M\right\} .
$$

We want to prove that $\mathcal{I}=\{1, \ldots, m\}$. Indeed, if this were not the case, by the irreducible character of the matrix $B\left(x_{0}\right)$ there would exist $i \in \mathcal{I}$ and $k \notin \mathcal{I}$ such that

$$
b_{i k}\left(x_{0}\right)<0 .
$$

We now make use of the method of doubling the variables to reach a contradiction. For every $\varepsilon>0$, we set

$$
\psi^{\varepsilon}(x, y)=v_{i}(x)-u_{i}(y)-\frac{d(x, y)^{2}}{2 \varepsilon^{2}}-\frac{d\left(x, x_{0}\right)^{2}}{2}, \quad x, y \in \mathbb{T}^{N} .
$$

Let $M_{\varepsilon}=\max _{\mathbb{T}^{N} \times \mathbb{T}^{N}} \psi_{\varepsilon}$ and denote by $\left(x_{\varepsilon}, y_{\varepsilon}\right)$ a point in $\mathbb{T}^{N} \times \mathbb{T}^{N}$ where such a maximum is achieved. By a standard argument in the theory of viscosity solution, see for instance Lemma 2.3 in [2], the following properties hold:

$$
x_{\varepsilon}, y_{\varepsilon} \rightarrow x_{0}, \quad \frac{d\left(x_{\varepsilon}, y_{\varepsilon}\right)}{\varepsilon} \rightarrow 0 \quad \text { as } \varepsilon \rightarrow 0
$$

Furthermore,

$$
p_{\varepsilon}^{\prime}:=\frac{x_{\varepsilon}-y_{\varepsilon}}{\varepsilon^{2}} \in D^{-} u_{i}\left(y_{\varepsilon}\right), \quad p_{\varepsilon}:=p_{\varepsilon}^{\prime}-\left(x_{\varepsilon}-x_{0}\right) \in D^{+} v_{i}\left(x_{\varepsilon}\right) \quad \text { for every } \varepsilon>0 .
$$

By the Lipschitz character of $v_{i}$ we derive that the vectors $\left\{p_{\varepsilon}: \varepsilon>0\right\}$ are equibounded, hence, up to subsequences and in view of the estimates (2.5), we infer

$$
p_{\varepsilon}, p_{\varepsilon}^{\prime} \rightarrow p_{0} \quad \text { as } \varepsilon \rightarrow 0
$$


for some vector $p_{0} \in \mathbb{R}^{N}$. We now use the fact that $\mathbf{v}$ and $\mathbf{u}$ are a sub and supersolution of (3.1), respectively, to get

$$
\begin{gathered}
H_{i}\left(x_{\varepsilon}, p_{\varepsilon}\right)+\left(B\left(x_{\varepsilon}\right) \mathbf{v}\left(x_{\varepsilon}\right)\right)_{i} \leqslant 0 \\
H_{i}\left(y_{\varepsilon}, p_{\varepsilon}^{\prime}\right)+\left(B\left(y_{\varepsilon}\right) \mathbf{u}\left(y_{\varepsilon}\right)\right)_{i} \geqslant 0 .
\end{gathered}
$$

By subtracting the above inequalities and by passing to the limit for $\varepsilon \rightarrow 0$ we end up with

$$
\left(B\left(x_{0}\right)\left(\mathbf{v}\left(x_{0}\right)-\mathbf{u}\left(x_{0}\right)\right)\right)_{i} \leqslant 0,
$$

that is, since $i \in \mathcal{I}$ and the matrix $B\left(x_{0}\right)$ is degenerate,

$$
M b_{i i}\left(x_{0}\right) \leqslant \sum_{j \neq i}\left|b_{i j}\left(x_{0}\right)\right|\left(v_{j}\left(x_{0}\right)-u_{j}\left(x_{0}\right)\right) \leqslant M \sum_{j \neq i}\left|b_{i j}\left(x_{0}\right)\right|=M b_{i i}\left(x_{0}\right) .
$$

Hence the above inequalities are equalities, in particular $v_{k}\left(x_{0}\right)-u_{k}\left(x_{0}\right)=M$ since $b_{i k}\left(x_{0}\right) \neq 0$, in contrast with the fact that $k \notin \mathcal{I}$.

Definition 2.4. For every $\mathbf{a} \in \mathbb{R}^{m}$, we denote by $\mathcal{H}(\mathbf{a})$ the set of subsolutions of the weakly coupled system (1.2). We will more simply write $\mathcal{H}(a)$ whenever $\mathbf{a}=a \mathbb{1}$ for some constant $a \in \mathbb{R}$.

Lemma 2.5. The sets $\mathcal{H}(\mathbf{a})$ are convex and closed in $\left(\mathrm{C}\left(\mathbb{T}^{N}\right)\right)^{m}$, and increasing with respect to the partial ordering on $\mathbb{R}^{m}$.

Proof. Convexity and monotonicity are straightforward. The fact that the $\mathcal{H}(\mathbf{a})$ are closed is a direct consequence of stability of viscosity subsolutions.

We now focus our attention to the case $\mathbf{a}=a \mathbb{1}$. As a direct consequence of the definition of the semigroup $\mathcal{S}(t)$, we get the following assertion:

Proposition 2.6. Let $a \in \mathbb{R}$ and $\mathbf{u} \in\left(\operatorname{Lip}\left(\mathbb{T}^{N}\right)\right)^{m}$. Then $\mathbf{u}$ is a viscosity solution of (1.2) with $\mathbf{a}=a \mathbb{1}$ if and only if

$$
\mathbf{u}=\mathcal{S}(t) \mathbf{u}+t a \mathbb{1} \quad \text { in } \mathbb{T}^{N} \quad \text { for every } t>0 .
$$

We have the following characterization:

Proposition 2.7. Let $a \in \mathbb{R}$ and $\mathbf{u} \in\left(\operatorname{Lip}\left(\mathbb{T}^{N}\right)\right)^{m}$. The following facts are equivalent:

(i) $\mathbf{u} \in \mathcal{H}(a)$;

(ii) the map $t \mapsto \mathcal{S}(t) \mathbf{u}+t a \mathbb{1}$ is non-decreasing on $[0,+\infty)$.

In particular, the sets $\mathcal{H}(a)$ are stable under the action of the semigroup $\mathcal{S}(t)$, in the sense that $\mathcal{S}(t)(\mathcal{H}(a)) \subset \mathcal{H}(a)$.

The proof of this proposition is rather technical and it is postponed to the Appendix A.

Definition 2.8. The critical value $c$ of the weakly coupled system (1.2) is defined as

$$
c=\inf \{a \in \mathbb{R}: \mathcal{H}(a) \neq \varnothing\} .
$$

The following holds:

Proposition 2.9. The critical value $c$ is finite and $\mathcal{H}(c) \neq \varnothing$. 
Proof. By the growth assumptions on the Hamiltonians $H_{i}$ it is easily seen that the function $\mathbf{u} \equiv(0, \ldots, 0)^{T}$ is a subsolution of (1.2) for $a_{0} \mathbb{1}$ with $a_{0} \in \mathbb{R}$ big enough.

Let us proceed to show that $c$ is finite valued and that $\mathcal{H}(c) \neq \varnothing$. Let $\left(a_{n}\right)_{n}$ be a decreasing sequence converging to $c$ and let $\mathbf{u}_{n} \in \mathcal{H}\left(c_{n}\right)$ for each $n \in \mathbb{N}$. Up to neglecting the first terms, we can assume that $a_{n} \leqslant a_{0}$ for every $n \in \mathbb{N}$. Arguing as in the proof of Proposition 2.2, we obtain that the following inequalities are satisfied in the viscosity sense:

$$
\mu_{i} \leqslant H_{i}\left(x, D u_{i}^{n}\right) \leqslant a_{n}+M_{a_{0}} \quad \text { in } \mathbb{T}^{N}
$$

for every $i \in\{1, \ldots, m\}$ and $n \in \mathbb{N}$, showing that $c$ is finite. We now exploit Proposition 2.2 ; by the monotonicity of the sets $\mathcal{H}(a)$ with respect to $a$, we infer that the functions $\mathbf{u}_{n}$ are equi-Lipschitz. Up to subtracting a vector of the form $k_{n} \mathbb{1}$ to each $\mathbf{u}_{n}$, we can furthermore assume that $u_{1}^{n}(0)=0$ for every $n \in \mathbb{N}$, yielding $\sup _{n}\left\|u_{1}^{n}\right\|_{\infty} \leqslant L$ for some $L \in \mathbb{R}$ by the equi-Lipschitz character of the sequence. Moreover,

$$
\left\|u_{j}^{n}-u_{1}^{n}\right\|_{\infty} \leqslant C_{a_{0}} \quad \text { for every } j \in\{1, \ldots, m\} \text { and } n \in \mathbb{N},
$$

yielding

$$
\left\|u_{j}^{n}\right\|_{\infty} \leqslant C_{a_{0}}+L \quad \text { for every } j \in\{1, \ldots, m\} \text { and } n \in \mathbb{N} .
$$

Up to subsequences, by the Arzela-Ascoli theorem, we infer that

$$
\mathbf{u}^{n} \rightrightarrows \mathbf{u} \quad \text { in } \mathbb{T}^{N}
$$

and $\mathbf{u} \in \mathcal{H}(c)$ by stability of the notion of viscosity subsolution.

We now proceed to show that a weakly coupled system of the kind (1.2) with $\mathbf{a}=a \mathbb{1}$ possesses solutions if and only if $a$ equals the critical value $c$.

We start with a preliminary result.

Proposition 2.10. Let $B(x)$ be a continuous irreducible coupling matrix on $\mathbb{T}^{N}$ and let us assume that $B(x)$ is invertible for every $x \in \mathbb{T}^{N}$. Let $\mathbf{v}, \mathbf{u} \in\left(\mathrm{C}\left(\mathbb{T}^{N}\right)\right)^{m}$ be, respectively, a sub and a supersolution of the weakly coupled system (1.2), for some $\mathbf{a} \in \mathbb{R}^{m}$. Then

$$
\mathbf{v}(x) \leqslant \mathbf{u}(x) \quad \text { for every } x \in \mathbb{T}^{N} .
$$

Proof. Arguing as in the proof of Proposition 2.2, we easily see that $\mathbf{v}$ is Lipschitz. Set

$$
M=\max _{1 \leqslant i \leqslant m} \max _{\mathbb{T}^{N}}\left(v_{i}-u_{i}\right) .
$$

We want to prove that $M \leqslant 0$. Assume by contradiction that $M>0$ and pick a point $x_{0} \in \mathbb{T}^{N}$ where such a maximum is attained. Set

$$
\mathcal{I}=\left\{i \in\{1, \ldots, m\}:\left(v_{i}\left(x_{0}\right)-u_{i}\left(x_{0}\right)\right)=M\right\} .
$$

Arguing as in the proof of Proposition 2.3 we infer that

$$
\left(B\left(x_{0}\right)\left(\mathbf{v}\left(x_{0}\right)-\mathbf{u}\left(x_{0}\right)\right)\right)_{i} \leqslant 0 \quad \text { for every } i \in \mathcal{I} .
$$

If $\mathcal{I}=\{1, \ldots, m\}$, inequality (2.7) is indeed an equality and this is in contradiction with the fact that $B\left(x_{0}\right)$ is invertible, in view of Proposition 1.3. If $\mathcal{I} \neq\{1, \ldots, m\}$, we choose $i \in \mathcal{I}$ and $k \notin \mathcal{I}$ such that $b_{i k}\left(x_{0}\right)<0$. From (2.7) and the assumption that $M>0$ we infer that

$$
M b_{i i}\left(x_{0}\right) \leqslant \sum_{j \neq i}\left|b_{i j}\left(x_{0}\right)\right|\left(v_{j}\left(x_{0}\right)-u_{j}\left(x_{0}\right)\right) \leqslant M \sum_{j \neq i}\left|b_{i j}\left(x_{0}\right)\right| \leqslant M b_{i i}\left(x_{0}\right),
$$


which implies that $v_{k}\left(x_{0}\right)-u_{k}\left(x_{0}\right)=M$, in contrast with the fact that $k \notin \mathcal{I}$.

The next result implies that solutions to a weakly coupled system of the kind (1.2) with $\mathbf{a}=a \mathbb{1}$ may exist only if $a$ equals the critical value.

Proposition 2.11. Let $a, b \in \mathbb{R}$ and $\mathbf{v}, \mathbf{u} \in\left(\mathrm{C}\left(\mathbb{T}^{N}\right)\right)^{m}$ such that the following inequalities are satisfied in the viscosity sense:

$$
\begin{array}{ll}
H_{i}\left(x, D v_{i}\right)+(B(x) \mathbf{v}(x))_{i} \leqslant a & \text { in } \mathbb{T}^{N} \\
H_{i}\left(x, D u_{i}\right)+(B(x) \mathbf{u}(x))_{i} \geqslant b & \text { in } \mathbb{T}^{N}
\end{array}
$$

for every $i \in\{1, \ldots, m\}$. Then $b \leqslant a$.

Proof. Let us assume by contradiction that $b>a$. Up to replacing $\mathbf{v}$ with $\mathbf{v}+k \mathbb{1}$ with $k>0$ big enough, we can assume

$$
\mathbf{v}>\mathbf{u} \quad \text { in } \mathbb{T}^{N}
$$

Let $\varepsilon>0$ such that $b-\varepsilon>a+\varepsilon$. By continuity of the functions $\mathbf{v}$ and $\mathbf{u}$, we can find $\lambda>0$ such that

$$
\left\|\lambda v_{i}\right\|_{\infty},\left\|\lambda u_{i}\right\|_{\infty}<\varepsilon \quad \text { for every } i \in\{1, \ldots, m\} .
$$

Then the following inequalities hold in the viscosity sense in $\mathbb{T}^{N}$ :

$H_{i}\left(x, D u_{i}\right)+((B(x)+\lambda \mathrm{I}) \mathbf{u}(x))_{i}>b-\varepsilon>a+\varepsilon>H_{i}\left(x, D v_{i}\right)+((B(x)+\lambda \mathrm{I}) \mathbf{v}(x))_{i}$.

For ever $x \in \mathbb{T}^{N}$, the matrix $B(x)+\lambda \mathrm{I}$ is irreducible, satisfies (C) and the sum of the elements of each of its rows is strictly positive, hence it is invertible in view of Proposition 1.3. By Proposition 2.10 we conclude that

$$
\mathbf{v} \leqslant \mathbf{u} \quad \text { in } \mathbb{T}^{N}
$$

achieving a contradiction.

We are now able to prove existence of solutions for the critical system, following the lines of Fathi [22]. This result has been already obtained in literature in similar settings by making use of the so called ergodic approximation, see [32, 7].

Theorem 2.12. There exists a function $\mathbf{u} \in \mathcal{H}(c)$ that solves the weakly coupled system

$$
H_{i}\left(x, D u_{i}\right)+(B(x) \mathbf{u}(x))_{i}=c \quad \text { in } \mathbb{T}^{N} \quad \text { for every } i \in\{1, \ldots, m\}
$$

in the viscosity sense.

Proof. We have already proved in Proposition 2.9 that $\mathcal{H}(c) \neq \varnothing$. Let us introduce the quotient space $\hat{\mathcal{H}}=\mathcal{H}(c) \backslash \mathbb{R} \mathbb{1}$, where we identify critical subsolutions that differ by a constant vector belonging to $\mathbb{R} \mathbb{1}$. Arguing as in the proof of Proposition 2.9 , it is easily seen that $\hat{\mathcal{H}}$ is compact for the topology of uniform convergence. Indeed, it is isomorphic to the subset of $\mathcal{H}(c)$ of subsolutions whose first component vanishes at the point $x=0$. Moreover, since the viscosity semigroup commutes with the addition of vectors of the form $\lambda \mathbb{1}$ and leaves $\mathcal{H}(c)$ stable, it induces a continuous semigroup, denoted $\hat{S}$, on $\hat{\mathcal{H}}$.

By the Schauder-Tychonoff fixed point theorem (see [14]), $\hat{S}$ possesses a fixed point, that is, there exists an element $\hat{\mathbf{u}} \in \hat{\mathcal{H}}$ such that

$$
\forall t \geqslant 0, \quad \hat{S}(t) \hat{\mathbf{u}}=\hat{\mathbf{u}} .
$$


Lifting these relations to $\mathcal{H}(c)$, we get

$$
\forall t \geqslant 0 \text { there exists } c_{t} \in \mathbb{R} \text { such that } \mathcal{S}(t) \mathbf{u}=\mathbf{u}+c_{t} \mathbb{1},
$$

where $\mathbf{u}$ is any element in the equivalence class of $\hat{\mathbf{u}}$. Since $\mathcal{S}$ is a semigroup, one readily realizes that the following relations are verified:

$$
c_{t+s}=c_{t}+c_{s} \quad \text { for every } t, s>0 .
$$

Since $t \mapsto \mathcal{S}(t) \mathbf{u}$ is continuous, we necessarily deduce that $c_{t}=-t \tilde{c}$ for all $t>0$ for some constant $\tilde{c} \in \mathbb{R}$.

The identity $\mathcal{S}(t) \mathbf{u}=\mathbf{u}-t \tilde{c} \mathbb{1}$, for all $t \geqslant 0$, implies that $\mathbf{u}$ is a viscosity solution of (2.8) with $\tilde{c}$ in place of $c$, see Proposition 2.6. But then $\tilde{c}=c$ in view of Proposition 2.11 and the statement is proved.

\section{The Aubry set}

In this section we start our qualitative analysis on the critical weakly coupled system, i.e. the system (1.2) with $\mathbf{a}=c \mathbb{1}$, where $c$ is defined via (2.6). From now

on we will always assume the critical value $c$ to be equal to 0 . This renormalization is always possible by replacing each $H_{i}$ with $H_{i}-c$. The critical weakly coupled system reads as

$$
H_{i}\left(x, D u_{i}\right)+(B(x) \mathbf{u}(x))_{i}=0 \quad \text { in } \mathbb{T}^{N} \quad \text { for every } i \in\{1, \ldots, m\} .
$$

Solutions, subsolutions and supersolutions of (3.1) will be termed critical in the sequel. The family of critical subsolutions, we recall, is denoted by $\mathcal{H}(0)$.

Our qualitative analysis on the critical weakly coupled system is based on the notion of Mañé matrix, defined in analogy with that of the Mañé potential.

Definition 3.1. For all $(x, y, i, j) \in \mathbb{T}^{N} \times \mathbb{T}^{N} \times\{1, \ldots, m\} \times\{1, \ldots, m\}$, we define

$$
\Phi_{i, j}(y, x)=\sup _{\mathbf{v} \in \mathcal{H}(0)} v_{i}(x)-v_{j}(y) .
$$

The following properties hold:

Proposition 3.2. The Mañé matrix verifies the following properties:

(i) it is everywhere finite and Lipschtiz continuous;

(ii) $\Phi \cdot, j(y, \cdot) \in \mathcal{H}(0) \quad$ for every $(y, j) \in \mathbb{T}^{N} \times\{1, \ldots, m\}$;

(iii) for every $(y, j) \in \mathbb{T}^{N} \times\{1, \ldots, m\}$ and $\mathbf{v} \in \mathcal{H}(0)$,

$$
\mathbf{v}-v_{j}(y) \mathbb{1} \leqslant \Phi \cdot, j(y, \cdot) \quad \text { in } \mathbb{T}^{N},
$$

namely $\Phi_{\cdot, j}(y, \cdot)$ is the maximal critical subsolution whose $j$-th component vanishes at $y$;

(iv) the entries of the Mañé matrix are linked by the following triangular inequality:

$$
\Phi_{i, k}(x, z) \leqslant \Phi_{j, k}(x, y)+\Phi_{i, j}(y, z)
$$

for every $i, j, k \in\{1, \ldots, m\}$ and $x, y, z \in \mathbb{T}^{N}$. 
Proof. The fact that the Mañé matrix is well defined directly follows from Proposition 2.2. Lipschitz continuity comes from the equi-Lipschitz character of critical subsolutions.

The second assertion comes from the fact that $\Phi_{\cdot, j}(y, \cdot)$ is, for every fixed $(j, y)$, a supremum of critical subsolutions, hence itself a critical subsolution by Proposition 1.7 .

The third point is a direct consequence of the definition.

The last point comes from the fact that $\Phi_{, j}(y, \cdot)$ is the greatest subsolution whose $j$-th component vanishes at $y$. Since $\Phi_{\cdot, k}(x, \cdot)-\Phi_{j, k}(x, y) \mathbb{1}$ is a subsolution whose $j$-th component vanishes at $y$ we obtain that

$$
\Phi_{\cdot, k}(x, \cdot)-\Phi_{j, k}(x, y) \mathbb{1} \leqslant \Phi_{\cdot, j}(y, \cdot),
$$

which is the triangular inequality to be proved.

As in the case of a single critical equation, the Mañé vectors are "almost" critical solutions, in the sense precised below:

Proposition 3.3. Let $y_{0} \in \mathbb{T}^{N}$ and $i_{0} \in\{1, \ldots, m\}$. Then the function $\mathbf{u}=$ $\Phi_{\cdot, i_{0}}\left(y_{0}, \cdot\right)$ satisfies

$$
H_{i_{0}}\left(x, D u_{i_{0}}\right)+(B(x) \mathbf{u}(x))_{i_{0}}=0 \quad \text { in } \mathbb{T}^{N} \backslash\left\{y_{0}\right\} .
$$

and

$$
H_{i}\left(x, D u_{i}\right)+(B(x) \mathbf{u}(x))_{i}=0 \quad \text { in } \mathbb{T}^{N} \quad \text { for every } i \neq i_{0}
$$

in the viscosity sense.

Proof. We argue by contradiction, following the classical argument of [22] for the classical Mañé potential.

Let $(i, y)$ be such that either $i \neq i_{0}$ or $y \neq y_{0}$. Let us assume that the viscosity supersolution condition is violated at $(i, y)$. This means that there exists a $C^{1}$ function $\psi$ such that $\psi(x) \leqslant \Phi_{i, i_{0}}\left(y_{0}, x\right)$ for all $x$, with equality if and only if $x=y$, and

$$
H_{i}(x, D \psi(y))+\left(B(y) \Phi_{\cdot, i_{0}}\left(y_{0}, y\right)\right)_{i}<0 .
$$

Since $\psi$ is $C^{1}$, and $B(\cdot)$ and $\Phi_{\cdot, i_{0}}\left(x_{0}, \cdot\right)$ are continuous, it is clear that this strict inequality continues to hold in a neighborhood of $y$. We infer that it is possible to find $\varepsilon>0$ small enough such that the function $w_{i}:=\max \left\{\Phi_{i, i_{0}}\left(y_{0}, \cdot\right), \psi+\varepsilon\right\}$ verifies

$$
H_{i}\left(x, D w_{i}(x)\right)+(B(x) \mathbf{w}(x))_{i} \leqslant 0 \quad \text { for a.e. } x \in \mathbb{T}^{N},
$$

where $\mathbf{w}$ is the vector whose $i$-th coordinate is $w_{i}$ and whose other coordinates are those of $\Phi_{\cdot, i_{0}}\left(y_{0}, \cdot\right)$. In the case when $i=i_{0}$ and $y \neq y_{0}$, we choose $\varepsilon>0$ small enough in such a way that $w_{i}\left(y_{0}\right)=\Phi_{i, i_{0}}\left(y_{0}, y_{0}\right)=0$. Moreover, for every $j \neq i$,

$$
H_{j}\left(x, D w_{j}(x)\right)+(B(x) \mathbf{w}(x))_{j} \leqslant 0 \quad \text { for a.e. } x \in \mathbb{T}^{N},
$$

as it is easily seen from the fact that $b_{j i}(\cdot) \leqslant 0$ in $\mathbb{T}^{N}$ and $w_{i} \geqslant \Phi_{i, i_{0}}\left(y_{0}, \cdot\right)$.

We have thus shown that $\mathbf{w}$ is a critical subsolution with $w_{i_{0}}\left(y_{0}\right)=0, \mathbf{w} \geqslant$ $\Phi_{i, i_{0}}\left(y_{0}, \cdot\right)$ and $\mathbf{w} \not \equiv \Phi_{i, i_{0}}\left(y_{0}, \cdot\right)$, thus contradicting the maximality of $\Phi_{i, i_{0}}\left(y_{0}, \cdot\right)$ amongst subsolutions whose $i_{0}$-th coordinate vanishes at $y_{0}$.

Next, we show a strong invariance property enjoyed by the rows of the Mañé matrix.

Proposition 3.4. Let $i, j \in\{1, \ldots, m\}$ and $y \in \mathbb{T}^{N}$. If $\Phi_{\cdot, i}(y, \cdot)$ is a critical solution on $\mathbb{T}^{N}$, then $\Phi_{\cdot, j}(y, \cdot)$ is too. 
Proof. Let us set $\mathbf{v}:=\Phi_{\cdot, j}(y, \cdot)$ and $\mathbf{u}:=\Phi_{\cdot, i}(y, \cdot)+\Phi_{i, j}(y, y) \mathbb{1}$. In view of Proposition 3.3, we only need to show that

$$
H_{j}(y, p)+(B(y) \mathbf{v}(y))_{j} \geqslant 0 \quad \text { for every } p \in D^{-} v_{j}(y) .
$$

According to Proposition 3.2, $\mathbf{v} \leqslant \mathbf{u}$ in $\mathbb{T}^{N}$ and $v_{i}(y)=u_{i}(y)$. The functions $\mathbf{v}$ and $\mathbf{u}$ being respectively a critical subsolution and a solution, we can apply Proposition 2.3 to infer that $\mathbf{v}(y)=\mathbf{u}(y)$. This also implies that $D^{-} v_{j}(y) \subseteq D^{-} u_{j}(y)$. Exploiting again the fact that $\mathbf{u}$ is a critical solution we finally get

$$
0 \leqslant H_{j}(y, p)+(B(y) \mathbf{u}(y))_{j}=H_{j}(y, p)+(B(y) \mathbf{v}(y))_{j} \quad \text { for every } p \in D^{-} v_{j}(y) .
$$

In view of the previous proposition, the following definition is well posed:

Definition 3.5. The Aubry set $\mathcal{A}$ for the weakly coupled system (3.1) is the set defined as

$$
\mathcal{A}=\left\{y \in \mathbb{T}^{N}: \Phi_{\cdot, i}(y, \cdot) \text { is a critical solution }\right\},
$$

where $i$ is any fixed index in $\{1, \ldots, m\}$.

By the continuity of the Mañé matrix and the stability of the notion of viscosity solution, it is easily seen that $\mathcal{A}$ is closed. The analysis we are about to present will show that the Aubry set is nonempty: as in the corresponding critical scalar case, we will see that $\mathcal{A}$ is the set where the obstruction to the existence of globally strict critical subsolutions concentrates.

Definition 3.6. Let $\mathbf{v} \in \mathcal{H}(0)$. We will say that $v_{i}$ is strict at $y \in \mathbb{T}^{N}$ if there exist an open neighborhood $V$ of $y$ and $\delta>0$ such that

$$
H_{i}\left(x, D v_{i}(x)\right)+(B(x) \mathbf{v}(x))_{i}<-\delta \quad \text { for a.e. } x \in V .
$$

We will say that $v_{i}$ is strict in an open subset $U$ of $\mathbb{T}^{N}$ if it is strict at $y$ for every $y \in U$.

We start by establishing an auxiliary result that will be needed in the sequel.

Lemma 3.7. Let $\mathbf{w} \in \mathcal{H}(0)$ such that $w_{i}$ is strict at $y \in \mathbb{T}^{N}$. Then there exists $\widetilde{\mathbf{w}} \in \mathcal{H}(0)$ such that $\widetilde{w}_{i}$ is $C^{\infty}$ and strict in a neighborhood of $y$.

Proof. By hypothesis, there exist $r>0$ and $\delta>0$ such that

$$
H_{i}\left(x, D w_{i}(x)\right)+(B(x) \mathbf{w}(x))_{i}<-\delta \quad \text { for a.e. } x \in B_{2 r}(y) .
$$

Let $\phi: \mathbb{T}^{N} \rightarrow[0,1]$ be a $C^{\infty}$-function, compactly supported in $B_{r}(y)$ and such that $\phi \equiv 1$ in $B_{r / 2}(y)$. Let us denote by $\kappa$ a Lispchitz constant for the critical subsolutions and by $\omega$ a continuity modulus of $H_{i}$ in $\mathbb{T}^{N} \times B_{R}$ for some fixed $R>\kappa+\|D \phi\|_{\infty}$. Let $\left(\rho_{n}\right)_{n}$ be a sequence of standard mollifiers on $\mathbb{R}^{N}$ and define

$$
\psi_{n}(x)=\left(\rho_{n} * w_{i}\right)(x)+\left\|\rho_{n} * w_{i}-w_{i}\right\|_{\infty}, \quad x \in \mathbb{T}^{N} .
$$

Note that $\psi_{n} \geqslant w_{i}$ in $\mathbb{T}^{N}$ for every $n \in \mathbb{N}$ and

$$
d_{n}:=\left\|\psi_{n}-w_{i}\right\|_{\infty} \rightarrow 0 \quad \text { as } n \rightarrow+\infty .
$$

Up to neglecting the first terms, we furthermore assume that all the $d_{n}$ are less than 1. For every $n \in \mathbb{N}$, we define a function $\widetilde{\mathbf{w}}^{n} \in\left(\operatorname{Lip}\left(\mathbb{T}^{N}\right)\right)^{m}$ by setting

$$
\widetilde{w}_{j}^{n}(x)=w_{j}(x) \quad \text { if } j \neq i, \quad \widetilde{w}_{i}^{n}(x)=\phi(x) \psi_{n}(x)+(1-\phi(x)) w_{i}(x)
$$


for every $x \in \mathbb{T}^{N}$. It is apparent by the definition that $\widetilde{w}_{i}^{n}$ is of class $C^{\infty}$ in $B_{r / 2}(y)$. Moreover the functions $\left(\widetilde{w}_{i}^{n}\right)_{n}$, and hence the $\left(\widetilde{\mathbf{w}}^{n}\right)_{n}$, are equi-Lipschitz. Indeed, for almost every $x \in \mathbb{T}^{N}$,

$$
D \widetilde{w}_{i}^{n}(x)=\phi(x) D \psi_{n}(x)+(1-\phi(x)) D w_{i}(x)+\left(\psi_{n}(x)-w_{i}(x)\right) D \phi(x)
$$

that is $\left\|D \widetilde{w}_{i}^{n}\right\|_{\infty} \leqslant \kappa+\|D \phi\|_{\infty}$. We want to show that $n$ can be chosen sufficiently large in such a way that $\widetilde{\mathbf{w}}^{n} \in \mathcal{H}(0)$ and

$$
H_{i}\left(x, D \widetilde{w}_{i}^{n}(x)\right)+\left(B(x) \widetilde{\mathbf{w}}^{n}(x)\right)_{i}<-\frac{2}{3} \delta \quad \text { for a.e. } x \in B_{r}(y) .
$$

We first note that, since $\widetilde{w}_{i}^{n} \geqslant w_{i}$ and $b_{j i} \leqslant 0$ in $\mathbb{T}^{N}$ for every $j \neq i$, we have

$$
H_{j}\left(x, D \widetilde{w}_{j}^{n}(x)\right)+\left(B(x) \widetilde{\mathbf{w}}^{n}(x)\right)_{j} \leqslant 0 \quad \text { in } \mathbb{T}^{N} \quad \text { for every } j \neq i .
$$

Moreover, since $\widetilde{\mathbf{w}}^{n}$ agrees with $\mathbf{w}$ outside $B_{r}(y)$, in order to show that $\widetilde{\mathbf{w}}^{n}$ satisfies (3.4) also for $j=i$, it will be enough, by the convexity of $H_{i}$, to prove (3.3).

To this aim, we start by noticing that

$$
H_{i}\left(x, D \widetilde{w}_{i}^{n}(x)\right) \leqslant \phi(x) H_{i}\left(x, D \psi_{n}(x)\right)+(1-\phi(x)) H_{i}\left(x, D w_{i}(x)\right)+\omega\left(d_{n}\|D \phi\|_{\infty}\right)
$$

for almost every $x \in \mathbb{T}^{N}$, in view of (3.2) and of the convexity of $H_{i}$. By Jensen's inequality, for every $n>1 / r$ and every $x \in B_{r}$ we have

$$
\begin{aligned}
H_{i}\left(x, D \psi_{n}(x)\right) & =H_{i}\left(x, \int_{B_{1 / n}} D w_{i}(x-y) \rho_{n}(y) \mathrm{d} y\right) \\
& \leqslant \int_{B_{1 / n}} H_{i}\left(x, D w_{i}(x-y)\right) \rho_{n}(y) \mathrm{d} y \\
& \leqslant \omega(1 / n)+\int_{B_{1 / n}} H_{i}\left(x-y, D w_{i}(x-y)\right) \rho_{n}(y) \mathrm{d} y \\
& \leqslant-\int_{B_{1 / n}}(B(x-y) \mathbf{w}(x-y))_{i} \rho_{n}(y) \mathrm{d} y-\delta+\omega(1 / n) \\
& \leqslant-\left(B(x) \widetilde{\mathbf{w}}^{n}(x)\right)_{i}-\delta+\omega(1 / n)+\varepsilon_{n},
\end{aligned}
$$

where

$$
\varepsilon_{n}:=\sup _{|z| \leqslant 1 / n}\left\|\left(B(\cdot+z) \mathbf{w}(\cdot+z)-B(\cdot) \widetilde{\mathbf{w}}^{n}(\cdot)\right)_{i}\right\|_{\infty} .
$$

Since $\widetilde{\mathbf{w}}^{n} \rightrightarrows \mathbf{w}$ in $\mathbb{T}^{N}$ and all these functions are equi-Lipschitz, it is easily seen that $\lim _{n} \varepsilon_{n}=0$. Furthermore

$$
H_{i}\left(x, D w_{i}(x)\right) \leqslant-\left(B(x) \widetilde{\mathbf{w}}^{n}(x)\right)_{i}-\delta+\varepsilon_{n} \quad \text { for a.e. } x \in B_{r}(y) .
$$

We now choose $n>1 / r$ sufficiently large such that

$$
\omega\left(d_{n}\|D \phi\|_{\infty}\right)+\omega(1 / n)+\varepsilon_{n}<\frac{\delta}{6}
$$

and plug (3.6) and (3.7) into (3.5) to finally get (3.3). The assertion follows by setting $\widetilde{\mathbf{w}}:=\widetilde{\mathbf{w}}^{n}$ for such an index $n$.

The next proposition shows that the $i$-th component of any critical subsolution fulfills the supersolution test on $\mathcal{A}$. 
Proposition 3.8. Let $y \in \mathcal{A}$. Then, for every $i \in\{1, \ldots, m\}$ and $\mathbf{w} \in \mathcal{H}(0)$,

$$
H_{i}(y, p)+(B(y) \mathbf{w}(y))_{i}=0 \quad \text { for every } p \in D^{-} w_{i}(y) .
$$

Proof. Pick $\mathbf{w} \in \mathcal{H}(0)$ and set $\mathbf{u}=\Phi_{\cdot, i}(y, \cdot)+w_{i}(y) \mathbb{1}$. According to Proposition 3.2, $\mathbf{w} \leqslant \mathbf{u}$ and, by definition of $\mathbf{u}, w_{i}(y)=u_{i}(y)$, in particular $D^{-} w_{i}(y) \subseteq D^{-} u_{i}(y)$. Now we exploit the fact that $\mathbf{u}$ and $\mathbf{w}$ are a critical solution and subsolution, respectively: from Proposition 2.3 we infer that $\mathbf{w}(y)=\mathbf{u}(y)$, while Proposition 1.6 implies

$$
0 \geqslant H_{i}(y, p)+(B(y) \mathbf{w}(y))_{i}=H_{i}(y, p)+(B(y) \mathbf{u}(y))_{i} \geqslant 0 \quad \forall p \in D^{-} w_{i}(y) .
$$

Hence all the inequalities must be equalities and the statement follows.

A converse of this result is given by the following

Proposition 3.9. Let $i \in\{1, \ldots, m\}$. The following facts are equivalent:

(i) $y \notin \mathcal{A}$;

(ii) there exists $\mathbf{w} \in \mathcal{H}(0)$ such that $w_{i}$ is strict at $y$.

Moreover, $w_{i}$ can be taken of class $C^{1}$ in a neighborhood of $y$.

Proof. Let us assume (i). Since $y \notin \mathcal{A}$, the supersolution test for $\Phi_{\cdot, i}(y, \cdot)$ is violated at $(i, y)$. This means that there exists a $C^{1}$ function $\psi$ such that $\psi(x) \leqslant \Phi_{i, i}(y, x)$ for all $x$, with equality if and only if $x=y$, and

$$
H_{i}(x, D \psi(y))+\left(B(y) \Phi_{\cdot, i}(y, y)\right)_{i}<0 .
$$

We define a function $\mathbf{w} \in\left(\operatorname{Lip}\left(\mathbb{T}^{N}\right)\right)^{m}$ by setting

$$
w_{i}(\cdot)=\max \left\{\Phi_{i, i}(y, \cdot), \psi+\varepsilon\right\}, \quad w_{j}(\cdot)=\Phi_{j, i}(y, \cdot) \quad \text { for } j \neq i .
$$

Arguing as in the proof of Proposition 3.3 we see that it is possible to choose $\varepsilon>0$ in a such a way that $\mathbf{w}$ is a critical subsolution. Moreover, since $w_{i}$ agrees with $\psi+\varepsilon$ in a neighborhood of $y$, there exist $\delta>0$ and an open neighborhood $W$ of $y$ such that $w_{i}$ is of class $C^{1}$ in $W$ and

$$
H_{i}\left(x, D w_{i}(x)\right)+(B(x) \mathbf{w}(x))_{i}<-\delta \quad \text { for every } x \in W .
$$

Conversely, let assume (ii). According to Lemma 3.7, there exists $\widetilde{\mathbf{w}} \in \mathcal{H}(0)$ such that $\widetilde{w}_{i}$ is smooth and strict in a neighborhood of $y$, in particular

$$
H_{i}\left(y, D \widetilde{w}_{i}(y)\right)+(B(y) \widetilde{\mathbf{w}}(y))_{i}<0 .
$$

In view of Proposition 3.8 we conclude that $y \notin \mathcal{A}$.

Remark 3.10. Proposition 3.8 expresses the fact, roughly speaking, that the $i_{-}$ th component of a critical subsolution cannot be strict at $y$. However, since the supersolution test (3.8) is void when $D^{-} u_{i}(y)$ is empty, this fact cannot be directly used to prove the equivalence stated in Proposition 3.9, This is the reason why we needed the regularization Lemma 3.7.

We proceed by proving a global version of the previous proposition. We give a definition first.

Definition 3.11. Let $\mathbf{v} \in \mathcal{H}(0)$. We will say that $\mathbf{v}$ is strict at $y$ if $v_{i}$ is strict at $y$ for every $i \in\{1, \ldots, m\}$. We will say that $\mathbf{v}$ is strict in an open subset $U$ of $\mathbb{T}^{N}$ if it is strict at $y$ for every $y \in U$. 
Theorem 3.12. There exists $\mathbf{v} \in \mathcal{H}(0)$ which is strict in $\mathbb{T}^{N} \backslash \mathcal{A}$. In particular, the Aubry set $\mathcal{A}$ is closed and nonempty.

Proof. Fix $i \in\{1, \ldots, m\}$. We first construct a critical subsolution $\mathbf{v}^{i}$ whose $i$-th component is strict in $\mathbb{T}^{N} \backslash \mathcal{A}$. According to Proposition 3.9, for every $y \in \mathbb{T}^{N} \backslash \mathcal{A}$ there exist an open neighborhood $W_{y}$ of $y$, a critical subsolution $\mathbf{w}^{y}$ and $\delta_{y}>0$ such that

$$
H_{i}\left(x, D w_{i}^{y}(x)\right)+\left(B(x) \mathbf{w}^{y}(x)\right)_{i}<-\delta_{y} \quad \text { for a.e. } x \in W_{y}
$$

The family $\left\{W_{y}: y \in \mathbb{T}^{N} \backslash \mathcal{A}\right\}$ is an open covering of $\mathbb{T}^{N} \backslash \mathcal{A}$, from which we can extract a countable covering $\left(W_{n}\right)_{n}$ of $\mathbb{T}^{N} \backslash \mathcal{A}$. For each $n \in \mathbb{N}$, let us denote by $\left(\mathbf{w}^{n}, \delta_{n}\right)$ the corresponding pair in $\mathcal{H}(0) \times(0,+\infty)$ that satisfies (3.9) in $W_{n}$. Up to subtracting to each critical subsolution $\mathbf{w}^{n}$ a vector of the form $k_{n} \mathbb{1}$, we can moreover assume that $w_{1}^{n}(0)=0$. Hence the functions $\mathbf{w}^{n}$ are componentwise equi-Lipschitz and equi-bounded in view of Proposition 2.2 , in particular the function

$$
\mathbf{v}^{i}(x)=\sum_{n=1}^{\infty} \frac{1}{2^{n}} \mathbf{w}^{n}(x), \quad x \in \mathbb{T}^{N}
$$

is well defined and belongs to $\left(\operatorname{Lip}\left(\mathbb{T}^{N}\right)\right)^{m}$. By convexity of the Hamiltonians, for almost every $x \in \mathbb{T}^{N}$ we get

$$
H_{i}\left(x, D v_{i}^{i}(x)\right)+\left(B(x) \mathbf{v}^{i}(x)\right)_{i} \leqslant \sum_{n=1}^{\infty} \frac{1}{2^{n}}\left(H_{i}\left(x, D w_{i}^{n}(x)\right)+\left(B(x) \mathbf{w}^{n}(x)\right)_{i}\right) \leqslant 0 .
$$

Moreover, the above inequalities hold with $-\delta_{k} / 2^{k}$ in place of 0 almost everywhere in $W_{k}$, for every $k \in \mathbb{N}$. This shows that $\mathbf{v}^{i}$ is a critical subsolution, strict in $\mathbb{T}^{N} \backslash \mathcal{A}$. Now set

$$
\mathbf{v}(x)=\sum_{i=1}^{m} \frac{1}{m} \mathbf{v}^{i}(x), \quad x \in \mathbb{T}^{N} .
$$

A similar argument shows that $\mathbf{v}$ is a critical subsolution that satisfies the assertion.

If $\mathcal{A}=\varnothing$, by compactness we would have $\mathcal{H}(-\delta) \neq \varnothing$ for some $\delta>0$, contradicting the definition of the critical value $c=0$.

In view of Proposition 3.9, we have the following characterization:

Theorem 3.13. Let $y \in \mathbb{T}^{N}$. The following are equivalent facts:

(i) $y \notin \mathcal{A}$;

(ii) there exists $\mathbf{w} \in \mathcal{H}(0)$ which is strict at $y$;

(iii) there exists $\mathbf{w} \in \mathcal{H}(0)$ and $i \in\{1, \ldots, m\}$ such that $w_{i}$ is strict at $y$.

We end this section by extending to weakly coupled systems a result which is well known in the case of a single critical equation.

Proposition 3.14. $\mathcal{A}=\bigcap_{\mathbf{w} \in \mathcal{H}(0)}\left\{y \in \mathbb{T}^{N}:(\mathcal{S}(t) \mathbf{w})(y)=\mathbf{w}(y) \quad\right.$ for every $\left.t>0\right\}$.

Proof. Let us denote by $\mathcal{A}^{\prime}$ the set appearing at the right-hand side of the above equality. Fix a point $y \in \mathcal{A}$ and let $\mathbf{w}$ be any critical subsolution. For every fixed index $i \in\{1, \ldots, m\}$, the function $\mathbf{u}^{i}=\Phi_{\cdot, i}(y, \cdot)+w_{i}(y) \mathbb{1}$ satisfies $\mathbf{w} \leqslant \mathbf{u}^{i}$ in $\mathbb{T}^{N}$ 
and $w_{i}(y)=u_{i}(y)$. Moreover, $\mathbf{u}^{i}$ is a critical solution, hence it is a fixed point for the semigroup $\mathcal{S}(t)$ by Proposition 2.6. By monotonicity of the semigroup, we have

$$
w_{i}(y) \leqslant(\mathcal{S}(t) \mathbf{w})_{i}(y) \leqslant\left(\mathcal{S}(t) \mathbf{u}^{i}\right)_{i}(y)=u_{i}^{i}(y) \text { for every } t>0,
$$

hence all the inequalities must be equalities, in particular $(\mathcal{S}(t) \mathbf{w})_{i}(y)=w_{i}(y)$ for every $t>0$. This being true for every $i \in\{1, \ldots, m\}$ and $\mathbf{w} \in \mathcal{H}(0)$, we conclude that $y \in \mathcal{A}^{\prime}$.

To prove the converse inclusion, pick $y \in \mathcal{A}^{\prime}$ and assume by contradiction that $y \notin \mathcal{A}$. Fix $i \in\{1, \ldots, m\}$ and take a critical subsolution $\mathbf{v}$ such that $v_{i}$ is of class $C^{1}$ and strict in a neighborhood of $y$, according to Proposition 3.9. By Proposition 2.7, the map $(t, x) \mapsto v_{i}(x)$ is a subtangent to $(\mathcal{S}(t) v)_{i}(x)$ at $\left(t_{0}, y\right)$ for every $t_{0}>0$ and since the latter is a solution of the evolutionary system (1.3) we get

$$
H_{i}\left(y, D v_{i}(y)\right)+\left(B(y) \mathcal{S}\left(t_{0}\right) \mathbf{v}(y)\right)_{i} \geqslant 0 .
$$

By sending $t_{0} \rightarrow 0^{+}$we get a contradiction with the fact that $v_{i}$ is strict at $y$.

\section{Regularization}

The aim of this section is to show how a strict critical subsolution can be regularized outside the Aubry set. In the case of a single critical equation, it is known that such procedure can be performed in such a way that the output is a strict critical subsolution which is, in addition, of class $C^{1}$ on the whole torus, see [4, 23, 24]. This result holds for Hamiltonians that are locally Lipschitz in $(x, p)$ and strictly convex in $p$ and the proof relies on the following two facts: first, any critical subsolution is differentiable on the Aubry set and, second, its gradient is independent of the specific subsolution chosen. This latter rigidity property holds for weakly couples systems too, as we will show at the end of the current section. What prevents us to extend to systems the existence of $C^{1}$ strict critical subsolutions is the lack of information on differentiability properties of critical subsolutions on the Aubry set.

We first deal with the regularization issue. The tools are not new and are mainly borrowed from [23, 24]. However, we provide a proof for the reader's convenience.

We start with a local regularization argument.

Lemma 4.1. Let $\mathbf{u} \in \mathcal{H}(0)$ and assume that, for some $r>0, \delta>0$ and $y \in \mathbb{T}^{N} \backslash \mathcal{A}$ and for every $i \in\{1, \ldots, m\}$,

$$
H_{i}\left(x, D u_{i}(x)\right)+(B(x) \mathbf{u}(x))_{i}<-\delta \quad \text { for a.e. } x \in B_{2 r}(y) .
$$

Then, for every $\varepsilon>0$, there exists $\mathbf{u}^{\varepsilon} \in \mathcal{H}(0)$ such that

(i) $\left\|\mathbf{u}^{\varepsilon}-\mathbf{u}\right\|_{\infty}<\varepsilon$;

(ii) $\quad \mathbf{u}^{\varepsilon}=\mathbf{u} \quad$ in $\mathbb{T}^{N} \backslash B_{r}(y)$;

(iii) $\quad \mathbf{u}^{\varepsilon}$ is of class $C^{\infty}$ in $B_{r / 2}(y)$ and satisfies

$$
H_{i}\left(x, D u_{i}^{\varepsilon}(x)\right)+\left(B(x) \mathbf{u}^{\varepsilon}(x)\right)_{i}<-\frac{2}{3} \delta \quad \text { for every } x \in B_{r / 2}(y) .
$$

Proof. Let $\phi: \mathbb{T}^{N} \rightarrow[0,1]$ be a $C^{\infty}$ function, compactly supported in $B_{r}(y)$ and such that $\phi \equiv 1$ in $B_{r / 2}(y)$. Let $\left(\rho_{n}\right)_{n}$ be a sequence of standard mollifiers on $\mathbb{R}^{N}$. For every $n \in \mathbb{N}$, we define a function $\mathbf{w}^{n} \in\left(\operatorname{Lip}\left(\mathbb{T}^{N}\right)\right)^{m}$ by setting

$w_{i}^{n}(x)=\phi(x)\left(\rho_{n} * u_{i}\right)(x)+(1-\phi(x)) u_{i}(x) \quad$ for every $x \in \mathbb{T}^{N}$ and $i \in\{1, \ldots, m\}$. 
It is apparent by the definition that $\mathbf{w}^{n}$ is of class $C^{\infty}$ in $B_{r / 2}(y)$ and agrees with $\mathbf{u}$ outside $B_{r}(y)$. Arguing as in the proof of Lemma 3.7, we see that it is possible to choose $n$ large enough in such a way that $\mathbf{w}^{n}$ is a critical subsolution and satisfies (4.1). Since $\mathbf{w}^{n} \rightrightarrows \mathbf{u}$ in $\mathbb{T}^{N}$, the assertion follows by setting $\mathbf{u}^{\varepsilon}:=\mathbf{w}^{n}$ for a sufficiently large $n$.

We now prove the announced regularization result.

Theorem 4.2. There exists a critical subsolution which is strict and $C^{\infty}$ in $\mathbb{T}^{n} \backslash \mathcal{A}$. More precisely, for every critical subsolution $\mathbf{v}$ which is strict in $\mathbb{T}^{N} \backslash \mathcal{A}$ and for every $\varepsilon>0$, there exists $\mathbf{v}^{\varepsilon} \in \mathcal{H}(0)$ such that

(i) $\left\|\mathbf{v}^{\varepsilon}-\mathbf{v}\right\|_{\infty}<\varepsilon ;$

(ii) $\mathbf{v}^{\varepsilon}=\mathbf{v}$ on $\mathcal{A}$;

(iii) $\quad \mathbf{v}^{\varepsilon}$ is $C^{\infty}$ and strict in $\mathbb{T}^{n} \backslash \mathcal{A}$.

Moreover, the set of such smooth and strict subsolutions is dense in $\mathcal{H}(0)$.

Proof. We first show how to regularize a subsolution which is strict outside the Aubry set. Let $\mathbf{v}$ be such a subsolution (given by Theorem [3.12) and fix $\varepsilon>0$. Since $\mathbf{v}$ is strict in $\mathbb{T}^{N} \backslash \mathcal{A}$, there exists a continuous and non-negative function $\delta: \mathbb{T}^{N} \rightarrow \mathbb{R}$ with $\delta^{-1}(\{0\})=\mathcal{A}$ such that

$$
H_{i}\left(x, D v_{i}\right)+(B(x) \mathbf{v}(x))_{i} \leqslant-\delta(x) \quad \text { in } \mathbb{T}^{N}
$$

for every $i \in\{1, \ldots, m\}$. Clearly, it is not restrictive to assume that the inequality $\delta(x) \leqslant \min \left\{\varepsilon / 2, d(x, \mathcal{A})^{2}\right\}$ holds for every $x \in \mathbb{T}^{N}$. In view of Lemma 4.1, we can find a locally finite covering $\left(U_{n}\right)_{n}$ of $\mathbb{T}^{N} \backslash \mathcal{A}$ by open sets compactly contained in $\mathbb{T}^{N} \backslash \mathcal{A}$ and a sequence $\left(\mathbf{u}^{n}\right)_{n}$ of critical subsolutions such that each $\mathbf{u}^{n}$ is $C^{\infty}$ in $U_{n}$ and satisfies

$$
\begin{array}{rr}
H_{i}\left(x, D u_{i}^{n}\right)+\left(B(x) \mathbf{u}^{n}(x)\right)_{i} \leqslant-\frac{2}{3} \delta(x) & \text { for every } x \in U_{n}, \\
\left|\mathbf{u}^{n}(x)-\mathbf{v}(x)\right| \leqslant \delta(x) & \text { for every } x \in \mathbb{T}^{N} .
\end{array}
$$

Set

$$
\delta_{n}:=\inf _{x \in U_{n}} \delta(x) \quad \text { for every } n \in \mathbb{N}
$$

and choose a sequence $\left(\eta_{n}\right)_{n}$ in $(0,1)$ such that, for every $x \in \mathbb{T}^{N}$ and $n \in \mathbb{N}$, the following holds:

$$
\left|H(x, p)-H\left(x, p^{\prime}\right)\right|<\frac{\delta_{n}}{6} \quad \text { for all } p, p^{\prime} \in B_{\kappa+1} \text { with }\left|p-p^{\prime}\right|<\eta_{n},
$$

where $\kappa$ denotes a common Lipschitz constant for the critical subsolutions, in particular for all the $\mathbf{u}^{n}$. Last, take a smooth partition of unity $\left(\varphi_{n}\right)_{n}$ subordinate to $\left(U_{n}\right)_{n}$ and choose the functions $\mathbf{u}^{n}$ in such a way that the quantities $\left\|\mathbf{u}^{n}-\mathbf{v}\right\|_{\infty}$, which can be be made as small as desired, satisfy

$$
\sum_{\substack{k \in \mathbb{N} \\ U_{k} \cap U_{n} \neq \varnothing}}\left\|\mathbf{u}^{k}-\mathbf{v}\right\|_{\infty}\left\|D \varphi_{k}\right\|_{\infty}<\eta_{n} \quad \text { for every } n \in \mathbb{N} .
$$

That is always possible since the covering $\left(U_{n}\right)_{n}$ is locally finite.

We now define $\mathbf{v}^{\varepsilon}: \mathbb{T}^{N} \rightarrow \mathbb{R}^{m}$ by setting

$$
\mathbf{v}^{\varepsilon}(x)=\sum_{n=1}^{\infty} \varphi_{n}(x) \mathbf{u}^{n}(x) \quad \text { in } \mathbb{T}^{N} \backslash \mathcal{A} \quad \text { and } \quad \mathbf{v}^{\varepsilon}(x)=\mathbf{v}(x) \quad \text { on } \mathcal{A} \text {. }
$$


By definition, $\mathbf{v}^{\varepsilon}$ satisfies assertion (ii) and is $C^{\infty}$ in $\mathbb{T}^{N} \backslash \mathcal{A}$. From (4.2) we infer that $\left|\mathbf{v}^{\varepsilon}(x)-\mathbf{v}(x)\right| \leqslant \delta(x)$ in $\mathbb{T}^{N} \backslash \mathcal{A}$, which shows at once that $\mathbf{v}^{\varepsilon}$ is continuous in $\mathbb{T}^{N}$ and that it satisfies assertion (i). Moreover, by taking into account (4.4) and the fact that $\sum D \varphi_{k} \equiv 0$, one obtains, for every $x \in U_{n}$ and $i \in\{1, \ldots, m\}$, that

$$
\left|D v_{i}^{\varepsilon}(x)-\sum_{\substack{k \in \mathbb{N} \\ U_{k} \cap U_{n} \neq \varnothing}} \varphi_{k}(x) D u_{i}^{k}(x)\right|=\left|\sum_{\substack{k \in \mathbb{N} \\ U_{k} \cap U_{n} \neq \varnothing}}\left(u_{i}^{k}(x)-v(x)\right) D \varphi_{k}(x)\right|<\eta_{n},
$$

in particular

$$
\left|D v_{i}^{\varepsilon}(x)\right| \leqslant \eta_{n}+\sum_{\substack{k \in \mathbb{N} \\ U_{k} \cap U_{n} \neq \varnothing}} \varphi_{k}(x)\left|D u_{i}^{k}(x)\right| \leqslant 1+\kappa .
$$

We infer that $\mathbf{v}^{\varepsilon}$ is Lipschitz-continuous in $\mathbb{T}^{N}$. In order to prove that $\mathbf{v}^{\varepsilon}$ is a critical subsolution and is strict in $\mathbb{T}^{N} \backslash \mathcal{A}$, it will be enough to show that

$$
H_{i}\left(x, D v_{i}^{\varepsilon}(x)\right)+\left(B(x) \mathbf{v}^{\varepsilon}(x)\right)_{i} \leqslant-\frac{\delta(x)}{2} \quad \text { for a.e. } x \in \mathbb{T}^{N},
$$

for all $i \in\{1, \ldots, m\}$.

Recall the Lipschitz functions $\mathbf{v}^{\varepsilon}$ and $\mathbf{v}$ coincide on the Aubry set. Setting $\mathbf{w}^{\varepsilon}=$ $\mathbf{v}^{\varepsilon}-\mathbf{v}$, we infer that if $x_{0} \in \mathcal{A}$, then $\left|\mathbf{w}^{\varepsilon}(x)-\mathbf{w}^{\varepsilon}\left(x_{0}\right)\right| \leqslant d(x, \mathcal{A})^{2} \leqslant d\left(x, x_{0}\right)^{2}$. Hence $\mathbf{w}^{\varepsilon}$ is differentiable on $\mathcal{A}$ with vanishing differential and $D \mathbf{v}^{\varepsilon}(x)=D \mathbf{v}(x)$ for almost every $x \in \mathcal{A}$. Hence, it suffices to establish the claim in the complementary of $\mathcal{A}$. To this aim, by recalling the definition of $\eta_{n}$ and by making use of (4.5) and of Jensen inequality, we get that, for every $x \in U_{n}$ and $i \in\{1, \ldots, m\}$,

$$
\begin{gathered}
H_{i}\left(x, D v_{i}^{\varepsilon}(x)\right)+\left(B(x) \mathbf{v}^{\varepsilon}(x)\right)_{i} \leqslant H_{i}\left(x, \sum_{\substack{k \in \mathbb{N} \\
U_{k} \cap U_{n} \neq \varnothing}} \varphi_{k}(x) D u_{i}^{k}(x)\right)+\frac{\delta_{n}}{6} \\
+\sum_{\substack{k \in \mathbb{N} \\
U_{k} \cap U_{n} \neq \varnothing}} \varphi_{k}(x)\left(B(x) \mathbf{u}^{k}(x)\right)_{i} \\
\leqslant \sum_{\substack{k \in \mathbb{N} \\
U_{k} \cap U_{n} \neq \varnothing}} \varphi_{k}(x)\left(H_{i}\left(x, D u_{i}^{k}(x)\right)+\left(B(x) \mathbf{u}^{k}(x)\right)_{i}\right)+\frac{\delta_{n}}{6} \\
<-\frac{2}{3} \delta(x)+\frac{\delta_{n}}{6} \leqslant-\frac{\delta(x)}{2} .
\end{gathered}
$$

This concludes the proof of the first part of the statement.

For the density, let $\mathbf{u}$ be any critical subsolution. Let $\mathbf{v}$ be a critical subsolution which is strict outside the Aubry set (whose existence is assured by Theorem 3.12). Then, for any $\lambda \in(0,1)$, the function $(1-\lambda) \mathbf{u}+\lambda \mathbf{v}$ is a subsolution which is strict outside the Aubry set. This subsolution can therefore be regularized using the above procedure, giving a subsolution w which is strict and smooth outside the Aubry set. Moreover, both these steps can be done in such a way that $\|\mathbf{u}-\mathbf{w}\|_{\infty}$ is as small as wanted. This establishes the density.

We now additionally assume the Hamiltonians $H_{i}$ to be strictly convex in $p$ and derive some further information on the behavior of Clarke's generalized gradients of the critical subsolutions on the Aubry set.

We start with a preliminary lemma. 
Lemma 4.3. Let $y \in \mathcal{A}$ and let $\mathbf{u}^{1}, \cdots, \mathbf{u}^{\ell}$ be critical subsolutions. Then, for all $i \in\{1, \ldots, m\}$,

$$
\bigcap_{k=1}^{\ell} \partial^{c} u_{i}^{k}(x) \neq \varnothing .
$$

Moreover, it contains a vector $p_{i}$ which is extremal for all the sets $\partial^{c} u_{i}^{k}(x)$ and which satisfies

$$
H_{i}\left(y, p_{i}\right)+\left(B(y) \mathbf{u}^{k}(y)\right)_{i}=0 \quad \text { for every } k \in\{1, \ldots, \ell\}
$$

Proof. Let $\mathbf{w}=\frac{1}{\ell} \sum_{k=1}^{\ell} \mathbf{u}^{k} \in \mathcal{H}(0)$ and let $p_{i} \in \partial^{c} w_{i}(y)$ be such that

$$
H_{i}(y, p)+(B(y) \mathbf{w}(y))_{i}=0 .
$$

Such a $p_{i}$ must exist because otherwise $w_{i}$ would be strict at $y$. Note that, by strict convexity of $H_{i}$, the vector $p_{i}$ must be an extremal point of $\partial^{c} w_{i}(x)$, hence it is a reachable gradient of $w_{i}$. Let $y_{n} \rightarrow y$ be such that $u_{i}^{k}$ is differentiable at $y_{n}$ for every $k \in\{1, \ldots, \ell\}$ and $n \in \mathbb{N}$, and

$$
D w_{i}\left(y_{n}\right)=\frac{1}{\ell} \sum_{k=1}^{\ell} D u_{i}^{k}\left(y_{n}\right) \rightarrow p_{i}
$$

Up to extraction of a subsequence, we can assume that $D u_{i}^{k}\left(y_{n}\right) \rightarrow q_{k}$ for all $k \in$ $\{1, \ldots, \ell\}$. Then one readily obtains, by Jensen's inequality, that

$$
0=H_{i}\left(y, p_{i}\right)+(B(y) \mathbf{w}(y))_{i} \leqslant \frac{1}{\ell} \sum_{k=1}^{\ell}\left(H_{i}\left(y, q_{k}\right)+\left(B(y) \mathbf{u}^{k}(y)\right)_{i}\right) \leqslant 0 .
$$

Therefore, all the inequalities $\frac{1}{\ell}\left(H_{i}\left(y, q_{k}\right)+\left(B(y) \mathbf{u}^{k}(y)\right)_{i}\right) \leqslant 0$ summing to an equality, we deduce, by strict convexity of $H_{i}$, that $q_{1}=\cdots=q_{l}=p$. Moreover, since

$$
H_{i}\left(y, q_{k}\right)+\left(B(y) \mathbf{u}^{k}(y)\right)_{i}=0 \quad \text { for every } k \in\{1, \ldots, \ell\},
$$

and because of the strict convexity of $H_{i}$, one sees that $p_{i}$ is extremal, and thus reachable, for all the $u_{i}^{k}$.

We now extend the previous result as follows:

Proposition 4.4. Let $y \in \mathcal{A}$. Then, for each $i \in\{1, \ldots, m\}$, there exists a vector $p_{i} \in \mathbb{R}^{N}$ which is a reachable gradient of $u_{i}$ at $y$ for every $\mathbf{u} \in \mathcal{H}(0)$ and which satisfies

$$
H_{i}\left(y, p_{i}\right)+(B(y) \mathbf{u}(y))_{i}=0 .
$$

Proof. For each critical subsolution $\mathbf{u}$, let us denote by $P_{i}^{\mathbf{u}}$ the set of reachable gradients $p$ of $u_{i}$ at $y$ that satisfy $H_{i}(y, p)+(B(y) \mathbf{u}(y))_{i}=0$. This set is not empty and compact. The proposition amounts to proving that

$$
\bigcap_{\mathbf{u} \in \mathcal{H}(0)} P_{i}^{\mathbf{u}} \neq \varnothing
$$

If this were not the case, by compactness we could extract a finite empty intersection. But this would violate the previous lemma. 


\section{Rigidity of the Aubry set and Comparison Principle}

In this section we establish some interesting properties of the Aubry set and a comparison principle for the critical system. As we will see, these results follow rather easily thanks to the information gathered so far.

We start with a remarkable rigidity phenomenon that takes place on the Aubry set.

Theorem 5.1. Let $y \in \mathcal{A}$ and $i \in\{1, \ldots, m\}$. Then

$$
\mathbf{v}(y)=\Phi_{\cdot, i}(y, y)+v_{i}(y) \mathbb{1} \quad \text { for every } \mathbf{v} \in \mathcal{H}(0) .
$$

In particular, $\mathbf{v}(y)-\mathbf{w}(y) \in \mathbb{R} \mathbb{1}$ for any $\mathbf{v}, \mathbf{w} \in \mathcal{H}(0)$.

Proof. Take $\mathbf{v} \in \mathcal{H}(0)$ and set $\mathbf{u}:=\Phi_{\cdot, i}(y, \cdot)+v_{i}(y) \mathbb{1}$. According to Proposition 3.2, $\mathbf{u}$ is a critical solution satisfying $\mathbf{v} \leqslant \mathbf{u}$ in $\mathbb{T}^{N}$ and $v_{i}(y)=u_{i}(y)$. By applying Proposition 2.3 with $x_{0}:=y$ we get the assertion.

Remark 5.2. On the other hand, the above property does not hold when $y \notin \mathcal{A}$. Indeed, the proof of Lemma 3.7 shows that any critical subsolution $\mathbf{v}$ which is strict at $y$ can be modified in such a way that the output is a critical subsolution all of whose components except one coincide at $y$ with those of $\mathbf{v}$.

We derive two corollaries:

Corollary 5.3. Let $y \in \mathcal{A}$. Then the matrix $\Phi(y, y)$ is antisymetric.

Proof. Apply the previous theorem to the weak KAM solution $\Phi_{\cdot, j}(y, \cdot)$ and get

$$
\Phi \cdot, j(y, y)=\Phi_{\cdot, i}(y, y)+\Phi_{i, j}(y, y) \mathbb{1} .
$$

In particular,

$$
0=\Phi_{j, j}(y, y)=\Phi_{j, i}(y, y)+\Phi_{i, j}(y, y)
$$

Corollary 5.4. Let $y \in \mathcal{A}$. Then the critical solutions $\Phi_{\cdot, j}(y, \cdot)$ differ by a constant function. More precisely:

$$
\Phi_{\cdot, i}(y, \cdot)=\Phi_{\cdot, j}(y, \cdot)+\Phi_{j, i}(y, y) \mathbb{1} .
$$

Proof. Let us apply the last point of Proposition 3.2 twice:

$$
\begin{aligned}
& \Phi_{k, i}(y, z) \leqslant \Phi_{j, i}(y, y)+\Phi_{k, j}(y, z), \\
& \Phi_{k, j}(y, z) \leqslant \Phi_{i, j}(y, y)+\Phi_{k, i}(y, z) .
\end{aligned}
$$

In particular, we obtain

$$
\Phi_{k, i}(y, z) \leqslant \Phi_{j, i}(y, y)+\Phi_{k, j}(y, z) \leqslant \Phi_{j, i}(y, y)+\Phi_{i, j}(y, y)+\Phi_{k, i}(y, z)=\Phi_{k, i}(y, z),
$$

thanks to the previous corollary. Therefore all inequalities are equalities and that gives the result.

Next, we derive a comparison principle for sub and supersolutions of the critical weakly coupled system (3.1) which generalizes to our setting an analogous result established in [7] for Hamiltonians of a special Eikonal form, see Subsection 6.1 for more details. In particular, we obtain that $\mathcal{A}$ is a uniqueness set for the critical system. 
Theorem 5.5. Let $\mathbf{v}, \mathbf{u} \in\left(\mathrm{C}\left(\mathbb{T}^{N}\right)\right)^{m}$ be a sub and a supersolution of the critical weakly coupled system (3.1), respectively. Assume that

$$
\text { for every } x \in \mathcal{A} \text { there exists } i \in\{1, \ldots, m\} \text { such that } v_{i}(x) \leqslant u_{i}(x) .
$$

Then

$$
\mathbf{v}(x) \leqslant \mathbf{u}(x) \quad \text { for every } x \in \mathbb{T}^{N} .
$$

In particular, two critical solutions that coincide on $\mathcal{A}$ coincide on the whole $\mathbb{T}^{N}$.

Remark 5.6. The above theorem also implies that two critical solutions $\mathbf{u}$ and $\mathbf{v}$ are actually the same if (5.1) holds with an equality. This is consistent with Theorem 5.1, which assures that this "boundary" condition amounts to requiring that $\mathbf{u}=\mathbf{v}$ on $\mathcal{A}$.

Proof. In view of the density result stated in Theorem 4.2, the critical subsolution $\mathbf{v}$ can be approximated from below by a sequence of critical subsolutions that are, in addition, smooth and strict outside $\mathcal{A}$. Indeed, just pick a sequence $\left(\mathbf{w}^{n}\right)_{n \in \mathbb{N}}$ such that $\left\|\mathbf{w}^{n}-\mathbf{v}\right\|_{\infty}<n^{-1}$ and then define $\mathbf{v}^{n}=\mathbf{w}_{n}-n^{-1} \mathbb{1}$ which then verifies $\mathbf{v}^{n} \leqslant \mathbf{v}$ and $\left\|\mathbf{v}^{n}-\mathbf{v}\right\|_{\infty}<2 n^{-1}$. Clearly, each element of the sequence still satisfies the boundary condition (5.1), hence it is enough to prove the statement by additionally assuming $\mathbf{v}$ smooth and strict in $\mathbb{T}^{N} \backslash \mathcal{A}$.

Let us set

$$
M:=\max _{1 \leqslant i \leqslant m} \max _{\mathbb{T}^{N}}\left(v_{i}-u_{i}\right)
$$

and pick a point $x_{0} \in \mathbb{T}^{N}$ where such a maximum is attained. By Proposition 2.3 we know that $\mathbf{v}\left(x_{0}\right)=\mathbf{u}\left(x_{0}\right)+M \mathbb{1}$. If $x_{0} \notin \mathcal{A}$, then $v_{1}$ would be a smooth subtangent to $u_{1}$ at $x_{0}$. The function $\mathbf{u}$ being a supersolution, we would have

$$
0 \leqslant H_{1}\left(x_{0}, D v_{1}\left(x_{0}\right)\right)+\left(B\left(x_{0}\right) \mathbf{u}(x)\right)_{1}=H_{1}\left(x_{0}, D v_{1}\left(x_{0}\right)\right)+\left(B\left(x_{0}\right) \mathbf{v}(x)\right)_{1},
$$

in contrast with the fact that $\mathbf{v}$ is strict in $\mathbb{T}^{N} \backslash \mathcal{A}$. Hence $x_{0} \in \mathcal{A}$ and by the hypothesis (5.1) we get $M \leqslant 0$, as it was to be proved.

Last, we show that the trace of any critical subsolution on the Aubry set can be extended to the whole torus in such a way that the output is a critical solution.

Theorem 5.7. For any $\mathbf{v} \in \mathcal{H}(0)$, there exists a unique critical solution $\mathbf{u}$ such that $\mathbf{u}=\mathbf{v}$ on $\mathcal{A}$.

Proof. The assertion is derived by setting

$$
u_{i}(x)=\sup _{t>0}(\mathcal{S}(t) \mathbf{v})_{i}(x) \quad \text { for every } x \in \mathbb{T}^{N} \text { and } i \in\{1, \ldots, m\} .
$$

Indeed, the functions $\{\mathcal{S}(t) \mathbf{v}: t>0\}$ are equi-Lipschitz and non-decreasing with respect to $t$ and satisfy $\mathcal{S}(t) \mathbf{v}=\mathbf{v}$ on $\mathcal{A}$ for every $t>0$ by Proposition 3.14. We infer that $\mathbf{u}$ is a vector valued, Lipschitz continuous function and $\mathcal{S}(t) \mathbf{v} \rightrightarrows \mathbf{u}$ in $\mathbb{T}^{N}$ as $t \rightarrow+\infty$. Last, $\mathbf{u}$ is a critical solution for it is a fixed point of the semigroup $\mathcal{S}(t)$.

\section{EXAMPLES}

The critical value and the Aubry set for a weakly coupled system of the kind studied in this paper have, in general, no connections with those of each Hamiltonian, considered individually. This happens also in simple situations, see Remark 6.1 below. In this section, we present some examples where more explicit results may be obtained for the critical value and for the Aubry set. 
6.1. The setting of [7]. The first example we propose corresponds to the setting considered in [7. Assume that all the Hamiltonians are of the form $H_{i}(x, p)=$ $F_{i}(x, p)-V_{i}(x)$, where:

(a) $F_{i}$ and $V_{i}$ take non-negative values;

(b) $F_{i}$ is convex and coercive in $p$;

(c) $F_{i}(x, 0)=0$ for all $x \in \mathbb{T}^{N}$ and $i \in\{1, \ldots, m\}$.

Furthermore, assume that

$$
\bigcap_{i=1}^{m} V_{i}^{-1}(\{0\}) \neq \varnothing .
$$

Under these hypotheses, we claim that the critical value is 0 (whatever the coupling matrix is) and that the Aubry set is nothing but

$$
\mathcal{A}=\bigcap_{i=1}^{m} V_{i}^{-1}(\{0\}) .
$$

Indeed, it is easily seen that the null function $\mathbf{u}^{0}$ always belongs to $\mathcal{H}(0)$ under the first set of hypotheses. Therefore, $\mathcal{H}(0) \neq \varnothing$ and the critical value verifies $c \leqslant 0$. To see that there is actually equality, consider a point $x_{0} \in \cap V_{i}^{-1}(\{0\})$ and any $\left(C^{1}\right)$ function $\mathbf{u}$. An easy consequence of Proposition 1.4 yields that $B\left(x_{0}\right) \mathbf{u}\left(x_{0}\right)$ must have a non-negative entry, say $i$, hence

$$
H_{i}\left(x_{0}, D u_{i}\left(x_{0}\right)\right)+\left(B\left(x_{0}\right) \mathbf{u}\left(x_{0}\right)\right)_{i}=F_{i}\left(x_{0}, D u_{i}\left(x_{0}\right)\right)+\left(B\left(x_{0}\right) \mathbf{u}\left(x_{0}\right)\right)_{i} \geqslant 0 .
$$

Therefore, $\mathbf{u}$ cannot belong to a $\mathcal{H}(-\varepsilon)$ for a positive $\varepsilon$. The same argument can be adapted in the viscosity sense for any (non necessarily $C^{1}$ ) function. Therefore 0 is the critical value.

To prove that $\cap V_{i}^{-1}(\{0\})$ is the Aubry set, first notice that, for every $y \notin$ $\cap V_{i}^{-1}(\{0\})$, there exists an index $j$ such that $V_{j}(y)>0$. Then the $j$-th component of the null function $\mathbf{u}^{0}$ is strict at $y$. In view of Theorem 3.13 we get the inclusion

$$
\mathcal{A} \subseteq \bigcap_{i=1}^{m} V_{i}^{-1}(\{0\})
$$

The opposite inclusion is obtained as previously. Take any $\mathbf{u} \in \mathcal{H}(0)$ and $x_{0} \in$ $\cap V_{i}^{-1}(\{0\})$. We will do as if $\mathbf{u}$ is differentiable at $x_{0}$, but the argument carries on in the general case using test functions and the viscosity subsolution property. At $x_{0}$ we must have

$$
F_{i}\left(x_{0}, D u_{i}\left(x_{0}\right)\right)+\left(B\left(x_{0}\right) \mathbf{u}\left(x_{0}\right)\right)_{i} \leqslant 0 \quad \text { for every } i \in\{1, \ldots, m\} .
$$

But this is only possible if $F_{i}\left(x_{0}, D u_{i}\left(x_{0}\right)\right)=0$ for all $i \in\{1, \ldots, m\}$ and $B\left(x_{0}\right) \mathbf{u}\left(x_{0}\right)=$ 0 . Indeed, otherwise, $B\left(x_{0}\right) \mathbf{u}\left(x_{0}\right)$ will have a positive entry in view of Proposition 1.4, which is impossible. In particular, the above inequality holds with an equality. Since this happens for any critical subsolution $\mathbf{u}$, we get $x_{0} \in \mathcal{A}$ in view of Theorem 3.13. As a byproduct, this also establishes that at any point of $\mathcal{A}$, any critical subsolution must take as value a vector belonging to $\mathbb{R} \mathbb{1}$. This is a particular case of Theorem 5.1 and accounts for the type of symmetries already remarked in [7] for the critical solutions obtained via the asymptotic procedure therein considered. 
Remark 6.1. It would be interesting to understand what the Aubry set is for the weakly coupled system considered in the previous example when condition (6.1) is dropped. Unfortunately, we are not able to give an answer to this question. Note that, in this case, $c<0$. Indeed, if $c$ were greater or equal than 0 , then the null function would satisfy condition (iii) in Theorem 3.13 at any point $y \in \mathbb{T}^{N}$, contradicting the fact that the Aubry set is nonempty. We point out that similar examples appear in [32, Remark 3.5] and [36, Example 1.2].

6.2. The setting of [7] revisited. This second example is taken from [36]: the Hamiltonians are still of the form $H_{i}(x, p)=F_{i}(x, p)-V_{i}(x)$, but the quantities $\lambda_{i}:=\min _{\mathbb{T}^{N}} V_{i}$ are not required to be zero. The analogous condition

$$
\bigcap_{i=1}^{m} V_{i}^{-1}\left(\left\{\lambda_{i}\right\}\right) \neq \varnothing
$$

is in force. Moreover, the coupling matrix is taken independent of $x$. We claim that

$$
c=-\pi(\boldsymbol{\lambda}) \quad \text { and } \quad \mathcal{A}=\bigcap_{i=1}^{m} V_{i}^{-1}\left(\left\{\lambda_{i}\right\}\right),
$$

where $\boldsymbol{\lambda}=\left(\lambda_{1}, \ldots, \lambda_{m}\right)$ and $\pi(\boldsymbol{\lambda})$ denotes the unique real number such that $\boldsymbol{\lambda}$ $\pi(\boldsymbol{\lambda}) \mathbb{1} \in \operatorname{Im}(B)$. Indeed, by replacing each $H_{i}$ with $H_{i}+\lambda_{i}$ we reduce to the case of Example 6.1 and we conclude by exploiting the following result:

Proposition 6.2. Let $H_{i}$ be convex and coercive Hamiltonians for every $i \in\{1, \ldots, m\}$ and assume that the coupling matrix is independent of $x$. For every $\boldsymbol{\lambda}=\left(\lambda_{1}, \ldots, \lambda_{m}\right)$, denote by $c_{\boldsymbol{\lambda}}$ and $\mathcal{A}_{\boldsymbol{\lambda}}$ the critical value and Aubry set of the weakly coupled system with $H_{i}-\lambda_{i}$ in place of $H_{i}$ for every $i \in\{1, \ldots, m\}$. Then

$$
c_{\boldsymbol{\lambda}}=c_{\mathbf{0}}-\pi(\boldsymbol{\lambda}) \quad \text { and } \quad \mathcal{A}_{\boldsymbol{\lambda}}=\mathcal{A}_{\mathbf{0}} \quad \text { for every } \boldsymbol{\lambda} \in \mathbb{R}^{m},
$$

where $\pi(\boldsymbol{\lambda})$ denotes the unique real number such that $\boldsymbol{\lambda}-\pi(\boldsymbol{\lambda}) \mathbb{1} \in \operatorname{Im}(B)$.

Proof. Fix $\boldsymbol{\lambda}=\left(\lambda_{1}, \ldots, \lambda_{m}\right) \in \mathbb{R}^{m}$. Then $\boldsymbol{\lambda}=\pi(\boldsymbol{\lambda}) \mathbb{1}+B \boldsymbol{\mu}$ for some $\boldsymbol{\mu} \in \mathbb{R}^{m}$ and for a unique scalar $\pi(\boldsymbol{\lambda})$, for $\mathbb{R}^{m} \cong \operatorname{Ker}(B) \oplus \operatorname{Im}(B)$ in view of the results of Section 1.2. If $\mathbf{u}$ is a solution of the critical weakly coupled system associated with $H_{1}, \ldots, H_{m}$, then $\mathbf{w}:=\mathbf{u}+\boldsymbol{\mu}$ is a solution of

$$
H_{i}\left(x, D w_{i}\right)-\lambda_{i}+(B \mathbf{w}(x))_{i}=c_{\mathbf{0}}-\pi(\boldsymbol{\lambda}) \quad \text { in } \mathbb{T}^{N} \quad \text { for every } i \in\{1, \ldots, m\},
$$

thus showing that $c_{\boldsymbol{\lambda}}=c_{\mathbf{0}}-\pi(\boldsymbol{\lambda})$. If we now take as $\mathbf{u}$ a subsolution of the critical weakly coupled system associated with $H_{1}, \ldots, H_{m}$ which is strict outside $\mathcal{A}_{\mathbf{0}}$, we easily see that $\mathbf{w}:=\mathbf{u}+\boldsymbol{\mu}$ is a subsolution of (6.2) which is strict outside $\mathcal{A}_{\mathbf{0}}$, thus showing $\mathcal{A}_{\boldsymbol{\lambda}} \subseteq \mathcal{A}_{\mathbf{0}}$. The reverse inclusion can be proved analogously. This concludes the proof.

6.3. Commuting Hamiltonians. In this last example we consider the case when the Hamiltonians are strictly convex and pairwise commute. If the Hamiltonians are of class $C^{1}$, that means

$$
\left\{H_{i}, H_{j}\right\}(x, p):=\left(\frac{\partial H_{i}}{\partial p} \frac{\partial H_{j}}{\partial x}-\frac{\partial H_{j}}{\partial p} \frac{\partial H_{i}}{\partial x}\right)(x, p)=0 \quad \text { in } \mathbb{T}^{N} \times \mathbb{R}^{N}
$$

for every $i, j \in\{1, \ldots, m\}$. If the Hamiltonians are only continuous, the commutation hypothesis must be expressed in terms of commutation of their Lax-Oleinik semigroup, see [13] for more details. We also make the additional assumption that, 
individually, all the Hamiltonians have 0 as critical value. Then, we claim that 0 is the critical value of the system as well (whatever the coupling is).

Indeed, it is proved in 13, 41, that the Hamiltonians have the same critical solutions. In particular, there exists a function $u \in \operatorname{Lip}\left(\mathbb{T}^{N}\right)$ satisfying

$$
H_{i}(x, D u)=0 \quad \text { in } \mathbb{T}^{N} \quad \text { for every } i \in\{1, \ldots, m\}
$$

in the viscosity sense. Since the coupling is degenerate, we infer that the function $\mathbf{u}^{0}=u \mathbb{1}$ is a solution of

$$
H_{i}\left(x, D u_{i}^{0}\right)+\left(B(x) \mathbf{u}^{0}(x)\right)_{i}=0 \quad \text { in } \mathbb{T}^{N} \quad \text { for every } i \in\{1, \ldots, m\} .
$$

Therefore, the claim is a direct consequence of Proposition 2.11. Moreover, in this setting, we may localize the Aubry set of the system using those of the individual Hamiltonians. In order to do so, let us recall another result from [13].

Theorem 6.3. Let $H_{1}, \cdots, H_{m}$ be pairwise commuting and strictly convex Hamiltonians, with common critical value equal to 0. Then they have the same Aubry set $\mathcal{A}^{*}$. Moreover, there exists a common critical subsolution $v$ which is smooth outside $\mathcal{A}^{*}$ and strict for each Hamiltonian, i.e.

$$
H_{i}(x, D v(x))<0 \quad \text { for every } x \in \mathbb{T}^{N} \backslash \mathcal{A}^{*} \text { and } i \in\{1, \ldots, m\} .
$$

Using this theorem, we easily see that the inclusion $\mathcal{A} \subseteq \mathcal{A}^{*}$ holds. Indeed, the function $\mathbf{v}(x):=v(x) \mathbb{1}$ is a critical subsolution for the system which is strict outside $\mathcal{A}^{*}$.

We also note that, as in the previous example, $\mathbf{u}(y) \in \mathbb{R} \mathbb{1}$ for every $y \in \mathcal{A}$ and every $\mathbf{u} \in \mathcal{H}(0)$ in view of Theorem 5.1 .

A particular case of this example is when all the $H_{i}$ are equal. In this case we get the more precise statement:

Proposition 6.4. Let $H$ be a convex Hamiltonian and assume $H_{1}=\cdots=H_{m}=H$. Then $\mathcal{A}=\mathcal{A}^{*}$. Moreover, all critical solutions of the system are of the form $\mathbf{u}=u \mathbb{1}$ where $u$ is a critical solution of $H$.

Proof. The inclusion $\mathcal{A} \subseteq \mathcal{A}^{*}$ can be proved arguing as above (note that we do not need the strict convexity assumption here). Let us prove the converse statement. Pick $\mathbf{v} \in \mathcal{H}(0)$ and set $v(x):=\max _{i} v_{i}(x)$ for every $x \in \mathbb{T}^{N}$. We claim that $v$ is a critical subsolution for $H$. Indeed, let $x \in \mathbb{T}^{N}$ and $p \in D^{+} v(x)$. Then $v(x)=v_{i}(x)$ for some $i \in\{1, \ldots, m\}$. Since $v \geqslant v_{i}$ with equality at $x$, we get $p \in D^{+} v_{i}(x)$. We now use the fact that $\mathbf{v}$ is a subsolution of the system to get

$$
H(x, p) \leqslant H(x, p)+(B(x) \mathbf{v}(x))_{i} \leqslant 0,
$$

where the first inequality comes from the fact that

$$
(B(x) \mathbf{v}(x))_{i}=\sum_{j=1}^{m} b_{i j}(x) v_{j}(x) \geqslant \sum_{j=1}^{m} b_{i j}(x) v(x)=0,
$$

which holds true since $b_{i j}(x) \leqslant 0$ and $v_{j}(x) \leqslant v(x)$ for every $j \neq i$. Let us now assume that $\mathbf{v}$ is strict outside $\mathcal{A}$. Then the right inequality in (6.3) is strict as soon as $x \notin \mathcal{A}$, yielding that $v$ is a subsolution for $H$ which strict in the complementary of $\mathcal{A}$. This proves that $\mathcal{A}^{*} \subseteq \mathcal{A}$, hence $\mathcal{A}=\mathcal{A}^{*}$.

Let now $\mathbf{u}$ be a critical solution for the system. Then $v(x):=\max _{i} v_{i}(x)$ is a critical subsolution for $H$. Moreover, as

$$
\mathbf{u}(x)=u_{1}(x) \mathbb{1} \quad \text { for every } x \in \mathcal{A},
$$


we deduce that $v=u_{1}$ on $\mathcal{A}$. Since $\mathcal{A}=\mathcal{A}^{*}$, there exists a critical solution $\tilde{u}$ for $H$ such that $\tilde{u}=v$ on $\mathcal{A}$. Now the function $\tilde{\mathbf{u}}=\tilde{u} \mathbb{1}$ is a critical solution of the weakly coupled system satisfying $\tilde{\mathbf{u}}=\mathbf{u}$ on $\mathcal{A}$. By the comparison principle, i.e. Theorem 5.5, we conclude that $\mathbf{u}=\tilde{\mathbf{u}}$.

\section{Appendix A.}

In this appendix we want to give a proof of Proposition 2.7.

In what follows, a function $u$ will be said to be semiconcave on an open subset $U$ of either $\mathbb{T}^{N}$ or $\mathbb{R}_{+} \times \mathbb{T}^{N}$ if, for every $x \in U$, there exists a vector $p_{x} \in \mathbb{R}^{N}$ such that

$$
u(y)-u(x) \leqslant\left\langle p_{x}, y-x\right\rangle+d(y, x) \omega(d(y, x)) \quad \text { for every } y \in U
$$

where $\omega$ is a modulus. It can be shown this is equivalent to requiring that for every $x, y \in U$ and $\lambda \in[0,1]$,

$$
\lambda u(x)+(1-\lambda) u(y) \leqslant u(\lambda x+(1-\lambda) y)+\lambda(1-\lambda) \omega(d(x, y)) .
$$

The vectors $p_{x}$ satisfying the above inequality are precisely the elements of $D^{+} u(x)$, which is thus always nonempty in $U$. Moreover, $\partial^{c} u(x)=D^{+} u(x)$ for every $x \in U$. By the upper semicontinuity of the map $x \mapsto \partial^{c} u(x)$ with respect to set inclusion, we get in particular that $D u$ is continuous in its domain of definition, see 8 .

We start with the following

Proposition A.1. Let $T>0$ and $G:[0, T] \times \mathbb{T}^{N} \times \mathbb{R}^{N} \rightarrow \mathbb{R}$ be a locally Lipschitz Hamiltonian such that $G(s, \cdot, \cdot)$ is a strictly convex Hamiltonian, for every fixed $s \in[0, T]$. Let $u(t, x)$ be a Lipschitz function in $[0, T] \times \mathbb{T}^{N}$ that solves the evolutive Hamilton-Jacobi equation

$$
\frac{\partial u}{\partial t}+G\left(t, x, D_{x} u\right)=0 \quad \text { in }(0, T) \times \mathbb{T}^{N}
$$

in the viscosity sense. Then

(i) for every $0<\tau<T$, the function $u$ is semiconcave in $[\tau, T) \times \mathbb{T}^{N}$;

(ii) if $u(0, \cdot)$ is semi-concave in $\mathbb{T}^{N}$, then the functions $\{u(t, \cdot): t \in[0, T)\}$ are equi-semiconcave.

Proof. Since $u$ is Lipschitz, up to modifying $G$ outside $[0, T] \times \mathbb{T}^{N} \times B_{R}$ for a sufficiently large $R>0$, we can assume that $G$ is superlinear in $p$, uniformly with respect to $(t, x)$. We are then in the setting considered by Cannarsa and Soner in [9] and item (i) follows from their results.

Let us prove (ii). Let us denote by $L(t, x, q)$ the the Lagrangian associated with $G$ through the Fenchel transform and by $u_{0}$ the initial datum $u(0, \cdot)$. It is well known, see for instance [8], that the following representation formula holds:

$$
u(t, x)=\inf _{\xi(t)=x}\left(u_{0}(\xi(0))+\int_{0}^{t} L(s, \xi(s), \dot{\xi}(s)) \mathrm{d} s\right), \quad(t, x) \in(0, T) \times \mathbb{T}^{N},
$$

where the infimum is taken by letting $\xi$ vary in the family of absolutely continuous curves from $[0, t]$ to $\mathbb{T}^{N}$. Moreover, the minimum is attained by some curve $\gamma$, which is, in addition, Lipschitz continuous (actually, of class $C^{1}$ ), see [11.

We claim that there exists a constant $\kappa$, only depending on $G$ and on the Lipschitz constant of $u$ in $[0, T] \times \mathbb{T}^{N}$, such that $\|\dot{\gamma}\|_{\infty} \leqslant \kappa$. To this aim, we apply Proposition 
2.4 in [28] to the function $u(t, x)$ and the curve $s \mapsto(s, \gamma(s))$ to get

$$
\frac{\mathrm{d}}{\mathrm{d} s} u(s, \gamma(s))=p_{t}(s)+\left\langle p_{x}(s), \dot{\gamma}(s)\right\rangle \quad \text { for a.e. } s \in[0, t],
$$

where $s \mapsto\left(p_{t}(s), p_{x}(s)\right)$ is a measurable and essentially bounded function on $[0, t]$ such that

$$
\left(p_{t}(s), p_{x}(s)\right) \in \partial^{c} u(s, \gamma(s)) \quad \text { for a.e. } s \in[0, t] .
$$

By integrating (A.3) and using the Fenchel inequality we get

$$
\begin{aligned}
u(t, x) & =u_{0}(\gamma(0))+\int_{0}^{t} p_{t}(s)+\left\langle p_{x}(s), \dot{\gamma}(s)\right\rangle \mathrm{d} s \\
& \leqslant u_{0}(\gamma(0))+\int_{0}^{t} p_{t}(s)+G\left(s, \gamma(s), p_{x}(s)\right)+L(s, \gamma(s), \dot{\gamma}(s)) \mathrm{d} s \\
& \leqslant u_{0}(\gamma(0))+\int_{0}^{t} L(s, \gamma(s), \dot{\gamma}(s)) \mathrm{d} s,
\end{aligned}
$$

where in the last inequality we used the fact that $u$ is a (sub)-solution of the time dependent equation, i.e.

$$
p_{t}+G\left(t, x, p_{x}\right) \leqslant 0 \quad \text { for every }\left(p_{t}, p_{x}\right) \in \partial^{c} u(t, x) \text { and }(t, x) \in(0, T) \times \mathbb{T}^{N} .
$$

Since $\gamma$ is minimizing, all the inequalities must be equalities, in particular we obtain

$$
\dot{\gamma}(s) \in \partial_{p} G\left(s, \gamma(s), p_{x}(s)\right) \quad \text { for a.e. } s \in[0, t] .
$$

This proves the claim by choosing

$$
\kappa:=\sup \left\{|q|: q \in \partial_{p} G(s, x, p),(s, x) \in[0, T] \times \mathbb{T}^{N},|p| \leqslant \operatorname{Lip}\left(u ;[0, T] \times \mathbb{T}^{N}\right)\right\},
$$

which is finite thanks to the convexity and the growth assumptions assumed on $G$ with respect to $p$.

Let us now fix $t \in(0, T), x_{1}, x_{2} \in \mathbb{T}^{N}, \lambda \in[0,1]$ and set $x=\lambda x_{1}+(1-\lambda) x_{2}$. Note that $x_{1}=x+(1-\lambda) h$ and $x_{2}=x-\lambda h$ for $h=x_{1}-x_{2}$. Let us denote by $\gamma$ a curve realizing the infimum in (A.2) for such a pair of $(t, x)$, by $K$ a Lipschitz constant for $L$ restricted to $[0, T] \times \mathbb{T}^{N} \times B(0,2 \kappa)$ and by $\omega$ a semi-concavity modulus for $u_{0}$. We get

$$
\begin{aligned}
\lambda u\left(t, x_{1}\right)+ & (1-\lambda) u\left(t, x_{2}\right)-u(t, x) \\
\leqslant & \lambda\left(u_{0}(\gamma(0)+(1-\lambda) h)+\int_{0}^{t} L(s, \gamma(s)+(1-\lambda) h, \dot{\gamma}(s)) \mathrm{d} s\right) \\
& +(1-\lambda)\left(u_{0}(\gamma(0)-\lambda h)+\int_{0}^{t} L(s, \gamma(s)-\lambda h, \dot{\gamma}(s)) \mathrm{d} s\right) \\
& -\left(u_{0}(\gamma(0))+\int_{0}^{t} L(s, \gamma(s), \dot{\gamma}(s)) \mathrm{d} s\right) \\
=\lambda & u_{0}(\gamma(0)+(1-\lambda) h)+(1-\lambda) u_{0}(\gamma(0)-\lambda h)-u_{0}(\gamma(0)) \\
& +\lambda\left(\int_{0}^{t}(L(s, \gamma(s)+(1-\lambda) h, \dot{\gamma}(s))-L(s, \gamma(s), \dot{\gamma}(s))) \mathrm{d} s\right. \\
& +(1-\lambda) \int_{0}^{t}(L(s, \gamma(s)-\lambda h, \dot{\gamma}(s))-L(s, \gamma(s), \dot{\gamma}(s))) \mathrm{d} s \\
\leqslant & \lambda(1-\lambda)\left(\omega\left(d\left(x_{1}, x_{2}\right)\right)+t K d\left(x_{1}, x_{2}\right)\right),
\end{aligned}
$$


which proves the assertion.

The result just proved will be applied to weakly coupled systems as follows:

Proposition A.2. Let $T>0$ and $\mathbf{u}=\left(u_{1}, \ldots, u_{m}\right) \in\left(\operatorname{Lip}\left([0, T] \times \mathbb{T}^{N}\right)\right)^{m}$ be a solution of the evolutionary weakly coupled system (1.3). Let $B(x)$ be Lipschitz and $H_{i}$ be locally Lipschitz and strictly convex, for some fixed index $i \in\{1, \ldots, m\}$. Then, for all $0<\tau<T$, the function $u_{i}$ restricted to $[\tau, T) \times \mathbb{T}^{N}$ is semiconcave. Moreover, if, the initial condition $u_{i}(0, \cdot)$ is semiconcave, then the functions $\left\{u_{i}(t, \cdot): t \in\right.$ $[0, T]\}$ are equi-semiconcave.

Proof. The function $u_{i}$ solves, for the given index $i \in\{1, \ldots, m\}$, a Hamilton-Jacobi equation of the kind (A.1) with

$$
G(t, x, p)=H_{i}(x, p)+(B(x) \mathbf{u}(t, x))_{i}, \quad(t, x, p) \in[0, T] \times \mathbb{T}^{N} \times \mathbb{R}^{N} .
$$

The conclusion follows by applying Proposition A.1.

We are now ready to prove Proposition 2.7 .

Proof of Proposition 2.7. We recall that, by convexity of the Hamiltonians, subsolutions to the critical system coincide with almost everywhere subsolutions. This fact will be repeatedly exploited along the proof.

Assume first that $t \mapsto \mathcal{S}(t) \mathbf{u}+t a \mathbb{1}$ is non-decreasing. Pick $t_{0}>0$ such that the $\operatorname{map}(t, x) \mapsto \mathcal{S}(t) \mathbf{u}(x)$ is differentiable at $\left(t_{0}, x\right)$ for almost every $x \in \mathbb{T}^{N}$ and

$$
\partial_{t} \mathcal{S}\left(t_{0}\right) \mathbf{u}(x) \geqslant-a \mathbb{1} \quad \text { for a.e. } x \in \mathbb{T}^{N} \text {. }
$$

By the Lipschitz character of the map $(t, x) \mapsto \mathcal{S}(t) \mathbf{u}(x)$ and Fubini's theorem, this holds true for almost every $t_{0}>0$. Using the evolutionary equation, which is verified at every differentiability point of $\mathcal{S}(t) \mathbf{u}(x)$, we deduce that, for every $i \in\{1, \ldots, m\}$,

$$
H_{i}\left(x, D\left(\mathcal{S}\left(t_{0}\right) \mathbf{u}\right)_{i}(x)\right)+\left(B(x) \mathcal{S}\left(t_{0}\right) \mathbf{u}(x)\right)_{i} \leqslant a \quad \text { for a.e. } x \in \mathbb{T}^{N},
$$

that is, $\mathcal{S}\left(t_{0}\right) \mathbf{u} \in \mathcal{H}(a)$. This being true for almost every $t_{0}>0$, the conclusion follows by stability of viscosity subsolutions.

Let us now assume reciprocally that $\mathbf{u} \in \mathcal{H}(a)$. We first approximate each Hamiltonian $H_{i}$ with a sequence $\left(H_{i}^{k}\right)_{k}$ of convex Hamiltonians that are, in addition, locally Lipschitz in $(x, p)$ and strictly convex in $p$. This can be done by taking a sequence $\left(\rho_{k}\right)_{k}$ of standard mollifiers on $\mathbb{R}^{N}$ and by setting

$$
H_{i}^{k}(x, p)=\int_{B_{1}} \rho_{k}(y) H_{i}(x-y, p) \mathrm{d} y+\frac{|p|^{2}}{k}, \quad(x, p) \in \mathbb{T}^{N} \times \mathbb{R}^{N} .
$$

Analogously, we approximate the matrix $B(x)$ by a sequence of coupling matrixes $\left(B_{k}(x)\right)_{k}$ that are Lipschitz in $x$. Note that, for each index $i \in\{1, \ldots, m\}, \quad H_{i}^{k} \rightrightarrows$ $H_{i} \quad$ in $\mathbb{T}^{N} \times \mathbb{R}^{N}$ and $B_{k} \rightrightarrows B$ in $\mathbb{T}^{N}$ as $k \rightarrow+\infty$. Let us denote by $\mathcal{H}_{k}(a)$ the set of $\mathbf{a}$-subsolution of the weakly coupled system (1.2) with $\mathbf{a}=a \mathbb{1}$ and with $H_{1}^{k}, \ldots, H_{m}^{k}$ and $B_{k}$ in place of $H_{1}, \ldots, H_{m}$ and $B$, respectively, and by $\mathcal{S}_{k}$ the semigroup associated with the corresponding time-dependent equation (1.3).

Next, we approximate $\mathbf{u}$ with a sequence of $\left(\mathbf{u}^{n}\right)_{n}$ of functions that are componentwise semi-concave by setting

$$
u_{i}^{n}(x)=\inf _{y \in \mathbb{T}^{N}} u_{i}(y)+n d(y, x)^{2} \quad \text { for every } x \in \mathbb{T}^{N} \text { and } i=1, \cdots, m .
$$


Fix $\varepsilon>0$. A standard argument shows that, for $n$ large enough, $\mathbf{u}^{n} \in \mathcal{H}(a+\varepsilon)$. Moreover, by the Lipschitz character of $\mathbf{u}^{n}$ and by the local uniform convergence of $\left(H_{1}^{k}, \ldots, H_{m}^{k}\right)$ to $\left(H_{1}, \ldots, H_{m}\right)$ and of $B_{k}$ to $B$, we also have that $\mathbf{u}^{n} \in \mathcal{H}_{k}(a+2 \varepsilon)$ for $k$ sufficiently large. We now apply Proposition A.2 to infer that the map $(t, x) \mapsto$ $\mathcal{S}_{k}(t) \mathbf{u}^{n}(x)$ is semiconcave in $[0, \tau] \times \mathbb{T}^{N}$ for every $\tau>0$. By using the fact that the gradient of a semiconcave function is continuous in its domain of definition and by choosing $\tau>0$ small enough, we get $\mathcal{S}_{k}(t) \mathbf{u}^{n} \in \mathcal{H}_{k}(a+3 \varepsilon)$ for every $0 \leqslant t \leqslant \tau$. By exploiting this information in the evolutive weakly coupled system, we get

$$
\frac{\partial}{\partial t} \mathcal{S}_{k}(t) \mathbf{u}^{n}(x) \geqslant-(a+3 \varepsilon) \mathbb{1} \quad \text { for a.e. }(t, x) \in(0, \tau) \times \mathbb{T}^{N},
$$

i.e.

$$
\mathcal{S}_{k}(t+h) \mathbf{u}^{n} \geqslant \mathcal{S}_{k}(t) \mathbf{u}^{n}-h(a+3 \varepsilon) \mathbb{1} \quad \text { for every } 0<t<t+h \leqslant \tau .
$$

Now, by the comparison principle for the evolution equation and by using the fact that the semigroup commutes with the addition of scalar multiples of the vector $\mathbb{1}$, we obtain that $t \mapsto \mathcal{S}_{k}(t) \mathbf{u}^{n}-t(a+3 \varepsilon) \mathbb{1}$ is non decreasing. We now exploit the fact that

$$
\mathcal{S}_{k}(t) \mathbf{u}^{n} \underset{k \rightarrow+\infty}{\rightrightarrows} \mathcal{S}(t) \mathbf{u}^{n} \quad \text { and } \quad \mathcal{S}(t) \mathbf{u}^{n} \underset{n \rightarrow+\infty}{\rightrightarrows} \mathcal{S}(t) \mathbf{u} \quad \text { in } \mathbb{R}_{+} \times \mathbb{T}^{N}
$$

to infer that

$$
t \mapsto \mathcal{S}(t) \mathbf{u}^{n}-t(a+3 \varepsilon) \mathbb{1} \quad \text { is non-decreasing on }[0,+\infty) .
$$

Being this true for every $\varepsilon>0$, we finally have that $t \mapsto \mathcal{S}(t) \mathbf{u}^{n}-t a \mathbb{1}$ is nondecreasing on $[0,+\infty)$.

The last assertion follows from the equivalence just proved, together with the fact that the semigroup $\mathcal{S}(t)$ is non-decreasing and commutes with addition of vectors of the form $a \mathbb{1}$ with $a \in \mathbb{R}$.

\section{REFERENCES}

[1] S. N. Armstrong And P. E. Souganidis, Stochastic homogenization of level-set convex Hamilton-Jacobi equations, Int. Math. Res. Not. IMRN, (2013), pp. 3420-3449.

[2] G. BARLes, Solutions de viscosité des équations de Hamilton-Jacobi, vol. 17 of Mathématiques \& Applications (Berlin) [Mathematics \& Applications], Springer-Verlag, Paris, 1994.

[3] G. Barles And P. E. Souganidis, On the large time behavior of solutions of Hamilton-Jacobi equations, SIAM J. Math. Anal., 31 (2000), pp. 925-939 (electronic).

[4] P. Bernard, Existence of $C^{1,1}$ critical sub-solutions of the Hamilton-Jacobi equation on compact manifolds, Ann. Sci. École Norm. Sup. (4), 40 (2007), pp. 445-452.

[5] F. Cagnetti, D. Gomes, and H. V. Tran, Adjoint methods for obstacle problems and weakly coupled systems of PDE, ESAIM Control Optim. Calc. Var., 19 (2013), pp. 754-779.

[6] F. Camilli, O. Ley, And P. LoReti, Homogenization of monotone systems of HamiltonJacobi equations, ESAIM Control Optim. Calc. Var., 16 (2010), pp. 58-76.

[7] F. Camilli, O. Ley, P. Loreti, And V. D. NGuyen, Large time behavior of weakly coupled systems of first-order Hamilton-Jacobi equations, NoDEA Nonlinear Differential Equations Appl., 19 (2012), pp. 719-749.

[8] P. Cannarsa and C. Sinestrari, Semiconcave functions, Hamilton-Jacobi equations, and optimal control, Progress in Nonlinear Differential Equations and their Applications, 58, Birkhäuser Boston Inc., Boston, MA, 2004.

[9] P. Cannarsa And H. M. Soner, Generalized one-sided estimates for solutions of HamiltonJacobi equations and applications, Nonlinear Anal., 13 (1989), pp. 305-323.

[10] F. H. Clarke, Optimization and nonsmooth analysis, Canadian Mathematical Society Series of Monographs and Advanced Texts, John Wiley \& Sons Inc., New York, 1983. A WileyInterscience Publication. 
[11] F. H. Clarke And R. B. Vinter, Regularity properties of solutions to the basic problem in the calculus of variations, Trans. Amer. Math. Soc., 289 (1985), pp. 73-98.

[12] A. Davini And A. Siconolfi, A generalized dynamical approach to the large time behavior of solutions of Hamilton-Jacobi equations, SIAM J. Math. Anal., 38 (2006), pp. 478-502 (electronic).

[13] A. Davini And M. Zavidovique, Weak KAM theory for nonregular commuting Hamiltonians, Discrete Contin. Dyn. Syst. Ser. B, 18 (2013), pp. 57-94.

[14] J. Dugundu, Topology, Allyn and Bacon Inc., Boston, Mass., 1966.

[15] H. Engler And S. M. Lenhart, Viscosity solutions for weakly coupled systems of HamiltonJacobi equations, Proc. London Math. Soc. (3), 63 (1991), pp. 212-240.

[16] L. C. Evans, Adjoint and compensated compactness methods for Hamilton-Jacobi PDE, Arch. Ration. Mech. Anal., 197 (2010), pp. 1053-1088.

[17] A. FAthi, Solutions KAM faibles conjuguées et barrières de Peierls, C. R. Acad. Sci. Paris Sér. I Math., 325 (1997), pp. 649-652.

[18] — Théorème KAM faible et théorie de Mather sur les systèmes lagrangiens, C. R. Acad. Sci. Paris Sér. I Math., 324 (1997), pp. 1043-1046.

[19] — Orbites hétéroclines et ensemble de Peierls, C. R. Acad. Sci. Paris Sér. I Math., 326 (1998), pp. 1213-1216.

[20] _ - Sur la convergence du semi-groupe de Lax-Oleinik, C. R. Acad. Sci. Paris Sér. I Math., 327 (1998), pp. 267-270.

[21] - Weak KAM from a PDE point of view: viscosity solutions of the Hamilton-Jacobi equation and Aubry set, Proc. Roy. Soc. Edinburgh Sect. A, 142 (2012), pp. 1193-1236.

[22] — Weak KAM Theorem in Lagrangian Dynamics, preliminary version 10, Lyon. unpublished, June 152008.

[23] A. FAthi And A. Siconolfi, Existence of $C^{1}$ critical subsolutions of the Hamilton-Jacobi equation, Invent. Math., 155 (2004), pp. 363-388.

[24] — PDE aspects of Aubry-Mather theory for quasiconvex Hamiltonians, Calc. Var. Partial Differential Equations, 22 (2005), pp. 185-228.

[25] B. J. FeHRMAn, Stochastic homogenization of monotone systems of viscous Hamilton-Jacobi equations with convex nonlinearities, SIAM J. Math. Anal., 45 (2013), pp. 2441-2476.

[26] W. H. Fleming And H. M. Soner, Controlled Markov processes and viscosity solutions, vol. 25 of Applications of Mathematics (New York), Springer-Verlag, New York, 1993.

[27] H. IsHII, Perron's method for monotone systems of second-order elliptic partial differential equations, Differential Integral Equations, 5 (1992), pp. 1-24.

[28] — Asymptotic solutions for large time of Hamilton-Jacobi equations in Euclidean $n$ space, Ann. Inst. H. Poincaré Anal. Non Linéaire, 25 (2008), pp. 231-266.

[29] H. Ishit AND S. KoIKe, Viscosity solutions for monotone systems of second-order elliptic PDEs, Comm. Partial Differential Equations, 16 (1991), pp. 1095-1128.

[30] S. M. LENHART, Viscosity solutions for weakly coupled systems of first-order partial differential equations, J. Math. Anal. Appl., 131 (1988), pp. 180-193.

[31] P.-L. Lions, G. Papanicolaou, and S. Varadhan, Homogenization of Hamilton-Jacobi equation. unpublished preprint, 1987.

[32] H. Mitake And H. V. TRAn, Remarks on the large time behavior of viscosity solutions of quasi-monotone weakly coupled systems of Hamilton-Jacobi equations, Asymptot. Anal., 77 (2012), pp. 43-70.

[33] H. Mitake And H. V. Tran, A dynamical approach to the large-time behavior of solutions to weakly coupled systems of Hamilton-Jacobi equations, J. Math. Pures Appl. (9), 101 (2014), pp. 76-93.

[34] H. Mitake And H. V. Tran, Homogenization of Weakly Coupled Systems of Hamilton-Jacobi Equations with Fast Switching Rates, Arch. Ration. Mech. Anal., 211 (2014), pp. 733-769.

[35] G. Namah and J.-M. Roquejoffre, Convergence to periodic fronts in a class of semilinear parabolic equations, NoDEA Nonlinear Differential Equations Appl., 4 (1997), pp. 521-536.

[36] V. NGuYen, Some results on the large-time behavior of weakly coupled systems of first-order Hamilton-Jacobi equations, Journal of Evolution Equations, (2013), pp. 1-33.

[37] F. Rezakhanlou and J. E. Tarver, Homogenization for stochastic Hamilton-Jacobi equations, Arch. Ration. Mech. Anal., 151 (2000), pp. 277-309. 
[38] J.-M. RoqUeJoffre, Convergence to steady states or periodic solutions in a class of HamiltonJacobi equations, J. Math. Pures Appl. (9), 80 (2001), pp. 85-104.

[39] P. E. Souganidis, Stochastic homogenization of Hamilton-Jacobi equations and some applications, Asymptot. Anal., 20 (1999), pp. 1-11.

[40] G. G. Yin AND Q. Zhang, Continuous-time Markov chains and applications, vol. 37 of Applications of Mathematics (New York), Springer-Verlag, New York, 1998. A singular perturbation approach.

[41] M. Zavidovique, Weak KAM for commuting Hamiltonians, Nonlinearity, 23 (2010), pp. 793808.

Dip. di Matematica, Sapienza Università di Roma, P.le Aldo Moro 2, 00185 Roma, ITALY

E-mail address: davini@mat.uniroma1.it

imJ (projet Analyse Algébrique), UPMC, 4, place Jussieu, Case 247, 75252 Paris CÉdex 5, France

E-mail address: zavidovique@math.jussieu.fr 